\title{
The dynamics of ship propulsion unit-large hull-water interactions
}

\author{
Jing Tang Xing ${ }^{\mathrm{a}, \mathrm{b}}$, Zhe Tian ${ }^{\mathrm{b}, \mathrm{a}}$, Xinping Yan ${ }^{\mathrm{b}, \mathrm{c}, *}$
}

${ }^{a}$ FSI Research Group, Faculty of Engineering and the Environment, University of Southampton, Southampton SO17 1BJ, UK.

${ }^{\mathrm{b}}$ School of Energy and Power Engineering, Wuhan University of Technology, Wuhan, 430063, China.

${ }^{\mathrm{c}}$ National Engineering Research Center for Water Transportation Safety (WTSC), MOST, 430063, China

* Corresponding author.

E-mail addresses: xpyan@whut.edu.cn (Xinping Yan, corresponding author), jtxing@ @oton.ac.uk (Jing Tang Xing), tianzhe@whut.edu.cn (Zhe Tian)

\begin{abstract}
This paper developed a generalised theory to model the dynamics of an integrated ship propulsion unit-large hull-water interaction system. The engine shaft unit, the hull structure are considered as two substructures and the water as a subdomain, of which the motions of each subsystem are governed by the fundamental laws in continuum mechanics, and on their interfaces, kinematical and dynamical conditions are satisfied. The integrated variational formulation is given, based on which the numerical equation is derived by using the mode summation approach. The shaft frequency and deformation factors are defined to study on its interactions with large hull and water in order to provide a mean for safety propulsion unit design in large ships. An example is given to illustrate the applications of the general theory presented in the paper. Some guidelines for dynamical designs of large ship hull propulsion system are suggested.
\end{abstract}

Key Words: Propulsion unit-hull-water interaction; Fluid-structure interaction; Sea wave excitations; Shaft frequency / deformation factors; Natural vibration; Dynamic response.

\section{Introduction}

With fast development of ship sizes, dynamic interactions between ship hulls and engine propulsion systems have been playing more and more roles for safety operations of ships. For the type of small ships, the hull deformations excited by wave loads have no obvious effects on the operation of engine 
propulsion shaft systems. However, for large ships, especially with very big length, the deformation of ship may seriously change the mounting position of its propulsion shaft system, so that it could not normally work (Murawski, 2005; Shi et al, 2010; Shaft alignment, 2000). The statistical researches reported that about 52.9\% ship operation fails during 1998-2004 were caused by engine propulsion system problems (Leontopoulos, 2006; The Swedish Club Highlights, 2005), of which some photos of broken parts of main propulsion system can be read in (Dymarski, 2009; Fonte et al, 2009). Based on this practical situation as well as very strong requirements of worldwide ocean transportations, designers and scientists have to put much attention into dynamic interactions between ship hull and main engine system (Moctar et al, 2005; Ogawa et al, 2011; Lu et al, 2010; Pouw, 2008) in order to get the safety operations of large ships. Recently, a review paper (Yan et al, 2013) presents more details on the dynamic interactions between the propulsion system and large ship structures. The discussed problems involve the torsional vibration and its bearing arrangement (Murawski et al, 2015; Tang et al, 2013; Roemen et al, 2009), the robust global sliding model controls (Li, 2015; Li, 2015; Li, 2013) and the modelling with simulations (Tian et al, 2014; Tu et al, 2014) of marine propulsion system. The methods used to deal with the problems are mainly by numerical analysis, such as finite element models and substructure approaches (Schulten, 2005; Jun, 1998) .

Ships move on the water, the integrated system is a fluid-structure interaction system (Newman, 1978), for which the water flows affect ship motions and its elastic deformation so that the deformation of the engine propulsion system mounted in / on the ships. Reversely, the motions of ships are also affecting water flows through wet interaction interfaces. Therefore, to predicate more accurate deformation of engine shaft and hull structure and more safely to arrange the engine system, investigations on an integrated water-hull-engine system interaction is necessary, for example, the reported marine structures-water interactions (Newman, 1979; Bishop et al, 1986; Xing, 2009; Deruieux, 2003), to cite but too more. Based on the developed fluid-structure interaction theory and computer code (Xing, 1995; Xing, 1995) which has been used to simulate many dynamic problems in marine engineering (Xing, 2006; Xiong, 2006; Tan, 2006; Xiong, 2006; Xing, 2007; Xing, 2008), this paper intends to propose an integrated ship propulsion unit-large hull-water interaction model to reveal dynamic effects of water-hull interactions on large ship engine propulsion system. 


\section{Governing equations of integrated interaction system}

Fig. 1 shows an integrated ship propulsion unit-large hull-water interaction system studied in this paper. This system consists of a flexible hull structure of mass density $\rho_{s}$, body force $\hat{F}_{i}$ and elastic tensor $E_{i j k l}$ within a domain $\Omega_{s}$ of boundary $S=S_{T} \cup S_{w} \cup \Sigma$ with its unit normal vector $v_{i}$, the sound speed of water $c$, body force $\hat{f}_{i}$ and mass density $\rho_{f}$ in a domain $\Omega_{f}$ of boundary $\Gamma=\Gamma_{f} \cup \Gamma_{w} \cup \Gamma_{p} \cup \Sigma \cup \Gamma_{\infty}$ with a unit normal vector $\eta_{i}$ and a ship propulsion unit $\Omega_{p}$ mounted on the hull by $\hat{I}$ journal bearings $B_{I},(I=1,2, \cdots, \hat{I})$. Cartesian coordinate system $o-x_{1} x_{2} x_{3}$, where the gravitational acceleration $g$ is along the negative direction of the coordinate axis $o-x_{3}$, is chosen as a reference frame to describe the dynamics of interaction system. A hull-Lagrange coordinate system $O-X_{1} X_{2} X_{3}$, of which the three coordinator vectors are parallel to the ones of the system $o-x_{1} x_{2} x_{3}$, is fixed at the mass centre $O\left(x_{10}, x_{20}, x_{30}\right)$ of the ship hull. A propulsion unit-Lagrange coordinate system $\hat{o}-Y_{1} Y_{2} Y_{3}$ is fixed at a suitable point chosen by users, such as its centre of mass $\hat{o}\left(X_{10}, X_{20}, X_{30}\right)$ of the central line of the propulsion shaft. The relationship between the hullcoordinate system $O-X_{1} X_{2} X_{3}$ and the system $\hat{o}-Y_{1} Y_{2} Y_{3}$ for the propulsion unit is defined by an orthogonal transformation matrix $\boldsymbol{\beta}$, of which the components $\beta_{i j}=\cos \left(Y_{i}, X_{j}\right)$, here $\left(Y_{i}, X_{j}\right)$ denotes the angle between axis $\hat{O}-Y_{i}$ and $O-X_{j}$. The system may be excited by external dynamical forces $\hat{F}_{i}, \hat{T}_{i}, \hat{f}_{i}, \hat{p}$ and ground acceleration $\hat{w}_{i}$. The Cartesian tensor notations (Fung, 1977) with subscripts $\mathrm{i}, \mathrm{j}, \mathrm{k}$ and $\mathrm{l}(=1,2,3)$ obeying the summation convention are used in this paper. For example, $u_{i}, v_{i}, w_{i}, e_{i j}$ and $\sigma_{i j}$ represent the displacement, velocity, acceleration vectors, strain and stress tensors in solid, respectively, $\mathrm{p}$ denotes the pressure in fluid, $p_{, t t}=\partial^{2} p / \partial t^{2}$, $u_{i, j}=\partial u_{i} / \partial x_{j}, v_{i}=\dot{u}_{i}=u_{i, t}=\partial u_{i} / \partial t, w_{i}=\dot{v}_{i}=\ddot{u}_{i}=u_{i, t t}=\partial^{2} u_{i} / \partial t^{2}$ and Kronecker delta $\delta_{i j}$ etc. 


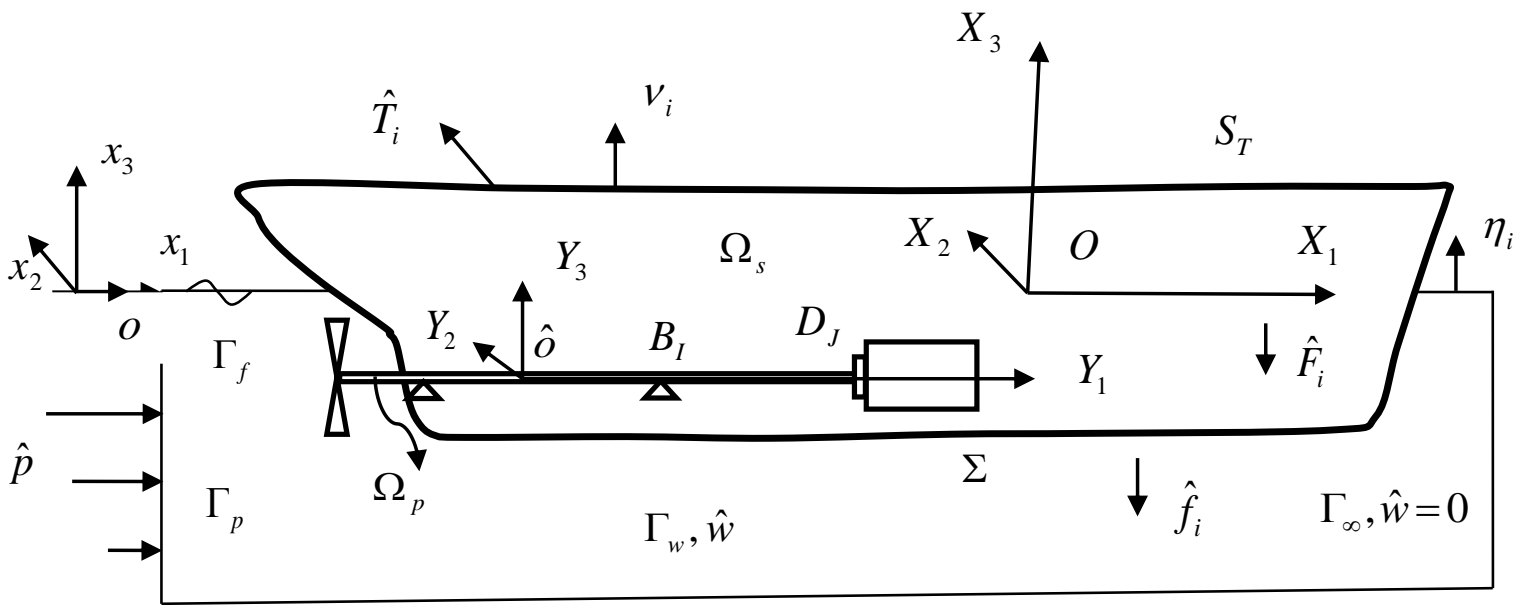

Fig. 1. The integrated ship propulsion unit-large hull-water interaction system

As shown in Fig. 2, we consider the propulsion unit as a shaft system consisting of the propeller $D_{p}$, supporting bearings $B_{I},(I=1,2, \cdots, \hat{I})$, engine crack box $D_{E}$ and attached disks $D_{J}, \quad(J=1,2, \cdots, \hat{J})$, to represent flying wheels, connectors. The shaft system $\hat{o}-Y_{1} Y_{2} Y_{3}$ is used to study the motion of this propulsion unit, which undergoes a translation and a rotation in / about axis $\hat{o}-Y_{1}$, two bending motions in the directions $\hat{o}-Y_{2}$ and $\hat{o}-Y_{3}$, respectively. We assume these motion components are governed by the classical beam, rod or shaft theory and their couplings are neglected. Therefore, the propulsion unit is represented by a shaft central line of mass density $\rho$ per unit volume, extension stiffness $E S$ and rotation stiffness $G J_{1}$ for axis $\hat{o}-Y_{1}$ as well as two bending stiffness $E J_{2}$ in the plane $Y_{1} \hat{o} Y_{2}$ and $E J_{3}$ the plane $Y_{1} \hat{o} Y_{3}$, respectively. A typical disk $D_{J}, \quad(J=1,2, \cdots, \hat{J})$, is fixed on the shaft line at point $\left(Y_{J 1}, Y_{J 2}=0=Y_{J 3}\right)$, which has concentrated mass $M_{J}$, inertial moment $I_{J 1}$ for rotation about $\hat{o}-Y_{1}$, inertial moment $I_{J 2}$ for bending in the plane $Y_{1} \hat{o} Y_{2}$ and $I_{J 3}$ for bending the plane $Y_{1} \hat{o} Y_{3}$.

We assume that the ship is in its stable equilibrium motion with a constant velocity on the water. The propulsion shaft unit has been mounted on the hull in a good alignment state (Shaft alignment, 2000; Leontopoulos, 2006). We are interested in the dynamic responses added on the equilibrium motion state of the system, which is caused by extra external forces, such as waves and earthquakes. Therefore, the coordinate system $o-x_{1} x_{2} x_{3}$ is considered as an inertial system in association with the 
ship constant velocity, which could be ignored. For a first instance to explore these complex dynamic interactions we consider the system is a linear system in which the hull motion is governed by linear elastic theory and the motion of the propulsion unit follows the beam / shaft theory as mentioned above. The water is compressible fluid with irrotational motions and a linear free surface wave condition. The dynamic pressure of the water satisfies a wave equation in the water domain. To derive the governing equations of the integrated interaction system, we have to model the dynamic interactions of the propulsion unit with the hull and the water through the bearings, engine crack box and the propeller, which is discussed and described by the corresponding equilibrium and geometrical relationships as follows.

\subsection{Propulsion unit-ship hull-water interactions}

\subsubsection{Bearings}

For a representative bearing $B_{I}$, of which the mass of moving parts is neglected, is fixed at a point $B_{I}\left(Y_{I 1}^{B}, Y_{I 2}^{B}, Y_{I 3}^{B}\right)$ in the hull and a point $A_{I}\left(Y_{I 1}^{A}, Y_{I 2}^{A}=0=Y_{I 3}^{A}\right)$ on the central line of the shaft. The coordinates of these two points in the hull system $O-X_{1} X_{2} X_{3}$ can be obtained from the following coordinate transformation

$$
X_{I i}^{A}=X_{i 0}+\beta_{j i} Y_{I j}^{A}, \quad X_{I i}^{B}=X_{i 0}+\beta_{j i} Y_{I j}^{B} .
$$

The interaction dynamic force components $\tilde{f}_{I i}^{A}$ and $\tilde{f}_{I i}^{B}$ at two ends of bearing $B_{I}$ in the shaft coordinate system, between the shaft and the hull can be calculated by using the stiffness coefficient $k_{l \hat{i}}$ (Bernhard Bettig, http://www.me.mtu.edu/ mdrl) but neglecting its damping $c_{I i}$ shown in Fig. 2, i.e.

$$
\left[\begin{array}{c}
\tilde{f}_{I i}^{A} \\
\tilde{f}_{I i}^{B}
\end{array}\right]=\left[\begin{array}{cc}
k_{l \hat{i}} & -k_{l \hat{i}} \\
-k_{l \hat{i}} & k_{l \hat{i}}
\end{array}\right]\left[\begin{array}{c}
U_{i}\left(Y_{I j}^{A}\right) \\
\beta_{i j} u_{j}\left(X_{I k}^{B}\right)
\end{array}\right], \quad\left[\begin{array}{l}
\tilde{f}_{I i}^{A s} \\
\tilde{f}_{I i}^{B h}
\end{array}\right]=-\left[\begin{array}{c}
\tilde{f}_{I i}^{A} \\
\tilde{f}_{I i}^{B}
\end{array}\right] \quad(\hat{i}=i),
$$

of which a positive value implies a pulling interaction force between two points $A_{I}$ and $B_{I}$. Due to interactions, the forces $\tilde{f}_{I i}^{A s}=-\tilde{f}_{I i}^{A}$ and $\tilde{f}_{I i}^{B h}=-\tilde{f}_{I i}^{B}$ are applied at the two corresponding points of the shaft and the hull, respectively. Here subscript $\hat{i}=i$ is introduced to avoid summation for tensor index. Here, $U_{i}$ denotes the shaft displacement in the system $\hat{o}-Y_{1} Y_{2} Y_{3}$ while $u_{i}$ represents the hull 
133 restricted.

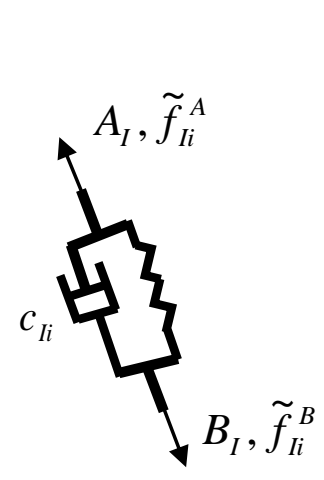

3

5

\subsubsection{Engine crack box} interactions,

displacement in the hull system $O-X_{1} X_{2} X_{3}$. Therefore, in Eq. (2), the hull displacement $u_{i}$ is premultiplied by the transformation tensor $\beta_{i j}$ to obtain its corresponding components in the system $\hat{o}-Y_{1} Y_{2} Y_{3}$. If the bearing parameter $k_{I 1} \rightarrow \infty$, it implies that the motion in direction $\hat{o}-Y_{1}$ is

Fig. 2. The propulsion unit: (a) journal bearing forces, stiffness and damping (but neglected in Eq. (2),
(b) the arrangement of shaft, propeller, bearings, disks as well as engine crack box.

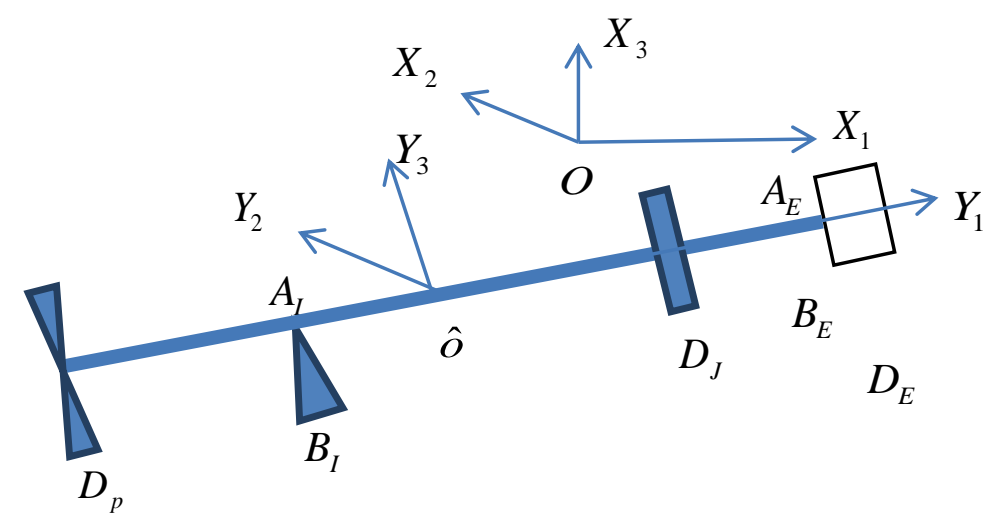

Fig. 2. The propulsion unit: (a) journal bearing forces, stiffness and damping (but neglected in Eq. (2),
(b) the arrangement of shaft, propeller, bearings, disks as well as engine crack box.

Fig. 2. The propulsion unit: (a) journal bearing forces, stiffness and damping (but neglected in Eq. (2),
(b) the arrangement of shaft, propeller, bearings, disks as well as engine crack box.

Similarly, the crank box $D_{E}$, of concentrated mass $M_{E}$ and three inertial moments $I_{E 1}, I_{E 2}$ and $I_{E 3}$, is fixed at point $B_{E}\left(Y_{E 1}^{B}, Y_{E 2}^{B}, Y_{E 3}^{B}\right)$ in the hull and point $A_{E}\left(Y_{E 1}^{A}, Y_{E 1}^{A}, Y_{E 1}^{A}\right)$ on the central line of the shaft. Replacing the subscript $I$ in Eqs. (1) and (2) by subscript $E$ to indicate the related variables or parameters of the crank box, we obtain the following equations for crank box-hull

$$
\begin{gathered}
X_{E i}^{A}=X_{i 0}+\beta_{j i} Y_{E j}^{A}, \quad X_{E i}^{B}=X_{i 0}+\beta_{j i} Y_{E j}^{B}, \\
{\left[\begin{array}{c}
\tilde{f}_{E i}^{A} \\
\tilde{f}_{E i}^{B}
\end{array}\right]=\left[\begin{array}{cc}
k_{E \hat{i}} & -k_{E \hat{i}} \\
-k_{E \hat{i}} & k_{E \hat{i}}
\end{array}\right]\left[\begin{array}{c}
U_{i}\left(Y_{E j}^{A}\right) \\
\beta_{i j} u_{j}\left(X_{E k}^{B}\right)
\end{array}\right], \quad\left[\begin{array}{l}
\tilde{f}_{E i}^{A s} \\
\tilde{f}_{E i}^{B h}
\end{array}\right]=-\left[\begin{array}{c}
\tilde{f}_{E i}^{A} \\
\tilde{f}_{E i}^{B}
\end{array}\right] \quad(\hat{i}=i),}
\end{gathered}
$$

As explained in Fig. 3, the engine moving parts produce a restoring moment $\tilde{m}_{E}$ about point $A_{E}$ to restrict the shaft rotation, which can be calculated by a rotation stiffness $k_{E}$ of engine in the form 


$$
\begin{aligned}
& \tilde{m}_{E}=k_{E} \theta_{1}\left(Y_{E 1}^{A}\right), \theta_{1}\left(Y_{E 1}^{A}\right)=\beta_{3 j}\left[u_{j}\left(X_{E i}^{B_{1}}\right)-u_{j}\left(X_{E i}^{B_{2}}\right)\right] /(2 d)=\Delta_{B} \boldsymbol{\beta}_{3} \mathbf{u}, \\
& \tilde{m}_{E}=d\left[\begin{array}{ll}
1 & -1
\end{array}\right]\left[\begin{array}{l}
\tilde{f}_{E 3}^{B_{1}} \\
\tilde{f}_{E 3}^{B_{2}}
\end{array}\right], \quad\left[\begin{array}{c}
\tilde{f}_{E 3}^{B_{1}} \\
\tilde{f}_{E 3}^{B_{2}}
\end{array}\right]=\frac{1}{4 d^{2}}\left[\begin{array}{cc}
k_{E} & -k_{E} \\
-k_{E} & k_{E}
\end{array}\right]\left[\begin{array}{l}
\beta_{3 j} u_{j}\left(X_{E i}^{B_{1}}\right) \\
\beta_{3 j} u_{j}\left(X_{E i}^{B_{2}}\right)
\end{array}\right], \quad\left[\begin{array}{l}
\tilde{f}_{E 3}^{B_{1 h}} \\
\tilde{f}_{E 3}^{B_{2 h}}
\end{array}\right]=-\left[\begin{array}{l}
\tilde{f}_{E 3}^{B_{1}} \\
\tilde{f}_{E 3}^{B_{2}}
\end{array}\right], \\
& \tilde{m}_{E}^{s}=-\tilde{m}_{E}=-k_{E} \Delta_{B} \boldsymbol{\beta}_{3} \mathbf{u}, \Delta_{B}=\left[\Delta\left(X_{i}-X_{E i}^{B_{1}}\right)-\Delta\left(X_{i}-X_{E i}^{B_{2}}\right)\right] /(2 d) . \quad \boldsymbol{\beta}_{3}=\left[\begin{array}{lll}
\beta_{31} & \beta_{32} & \beta_{33}
\end{array}\right]
\end{aligned}
$$

$148 \quad$ Here the two forces $\tilde{f}_{E 3}^{m}$ corresponding to the moment $\tilde{m}_{E}$ is added at the two hull points $B_{1}$ and $B_{2}$

of distance $2 d$ along direction $\hat{o}-Y_{3}$. The coordinates of these two points are given by

$$
\begin{array}{lc}
Y_{E i}^{B_{1}}=Y_{E i}^{B}+\delta_{i 2} d, & Y_{E i}^{B_{2}}=Y_{E i}^{B}-\delta_{i 2} d, \\
X_{E i}^{B_{1}}=X_{i 0}+\beta_{j i} Y_{E j}^{B_{1}}, & X_{E i}^{B_{2}}=X_{i 0}+\beta_{j i} Y_{E j}^{B_{2}} .
\end{array}
$$

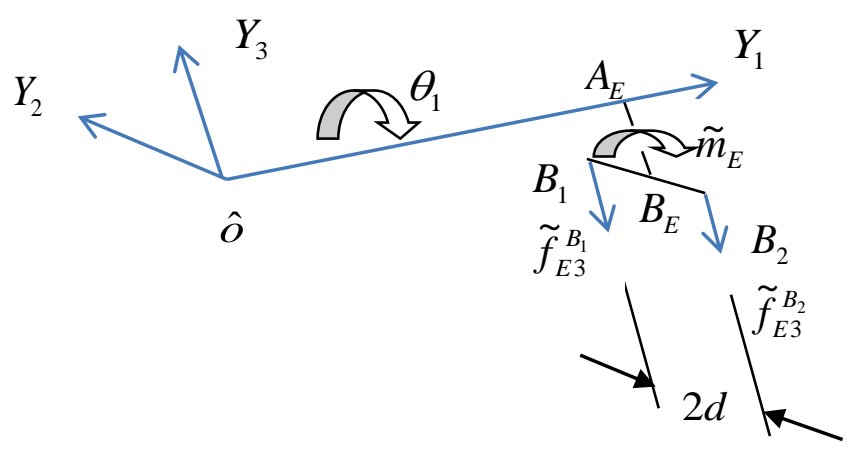

151

152

153

Fig. 3. The rotation angle $\theta_{1}$ and moment $\tilde{m}_{E}$, produced by the engine torsion stiffness, a moment $-\tilde{m}_{E}$ is applied at point $A_{E}$ of shaft. The hull provides a pair of forces $\tilde{f}_{E 3}^{B_{1}}=-\tilde{f}_{E 3}^{B_{2}}$ parallel to $\hat{o}-Y_{3}$ and applied at two engine points $B_{1}$ and $B_{2}$ of distance $2 d$ along direction $\hat{o}-Y_{3}$.

\subsubsection{Propeller}

The propeller $D_{p}$ is fixed at point $\left(Y_{p 1}, Y_{p 2}=0=Y_{p 3}\right)$ and has concentrated mass $M_{p}$ and three inertial moments $I_{p 1}, I_{p 2}$ and $I_{p 3}$ defined as same as the ones for disk $D_{J}$. Due to water-propeller interactions, the dynamic water forces $\tilde{f}_{p i}^{s}$ are added on the propeller shaft, which could be neglected if it is compared with the forces applied to the hull wet surface. 


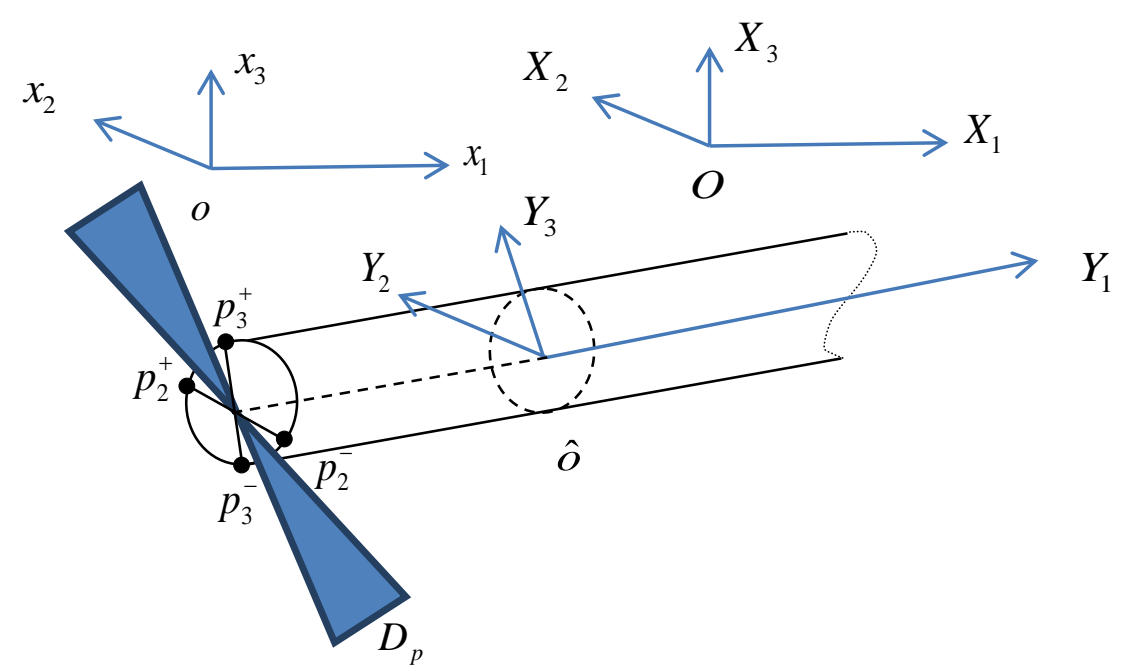

160

161

162

163

164

165

166

167

168

169

171

172

173

175

176

Fig. 4. The interaction forces between water and propeller shaft.

However, if it is to be considered, these forces are approximately estimated as follows. As shown in Fig. 4, in $Y_{1}-\hat{o}-Y_{2}$ plane, the difference of water pressure between $p_{2}^{+}$and $p_{2}^{-}$along $\hat{o}-Y_{2}$ is $p_{2}^{+}-p_{2}^{-}=\tilde{p}_{2}$, so that the interaction force can be calculated through multiplying $\tilde{p}_{2}$ by an effective area $A_{p 2}=D \times L=A_{p 3}$ in the system the system $\hat{o}-Y_{1} Y_{2} Y_{3}$, where the shaft diameter $D$ and wet part length $L$ are used. Therefore, the three interaction force components are represented by

$$
\tilde{f}_{p i}^{s}=-\tilde{f}_{p i}, \quad \tilde{f}_{p i}=\tilde{p} A_{p i}=\left(p_{\hat{i}}^{+}-p_{\hat{i}}^{-}\right) A_{p i}, \quad x_{p j}=x_{j 0}+X_{j 0}+\beta_{l j} Y_{p l}, \hat{i}=i .
$$

Considering the propeller effect in direction $\hat{o}-Y_{1}$, we introduce a coefficient $\alpha$ and take $A_{p 1}=\alpha A_{p 2}$

\subsection{Ship hull}

Using the interaction forces between the propulsion unit and the hull given in section 2.1, and linear theory of elasticity, we can now derive the governing equations describing the hull dynamics in this interaction system as follows.

Dynamic equation

$$
\begin{aligned}
& \sigma_{i j, j}+\hat{F}_{i}+\sum_{I=1}^{\hat{I}} \tilde{f}_{I j}^{B h} \beta_{j i} \Delta\left(X_{k}-X_{I k}^{B}\right)+\tilde{f}_{E j}^{B h} \beta_{j i} \Delta\left(X_{k}-X_{E k}^{B}\right) \\
& +\beta_{3 i}\left[\tilde{f}_{E 3}^{B_{1 h}} \Delta\left(X_{k}-X_{E k}^{B_{1}}\right)+\tilde{f}_{E 3}^{B_{2 h}} \Delta\left(X_{k}-X_{E k}^{B_{2}}\right)\right]=\rho_{s} w_{i}, \quad\left(X_{i}, t\right) \in \Omega_{s} \times\left(t_{1}, t_{2}\right),
\end{aligned}
$$


177 where $\Delta()$ denotes Delta function. The forces $\tilde{f}_{I j}^{B}, \tilde{f}_{E j}^{B}$ and $\tilde{f}_{E 3}^{m}$ defined in the shaft system are

178 transformed into the components in the hull system using the tensor $\beta_{i j}$, respectively.

179 Strain-displacement

180

$$
e_{i j}=\frac{1}{2}\left(u_{i, j}+u_{j, i}\right), \quad\left(X_{i}, t\right) \in \Omega_{s} \times\left(t_{1}, t_{2}\right) .
$$

181 Constitutive equation

$$
\sigma_{i j}=E_{i j k l} e_{k l}, \quad\left(X_{i}, t\right) \in \Omega_{s} \times\left(t_{1}, t_{2}\right) .
$$

183

and we have

$$
v_{i}=u_{i, t}, \quad w_{i}=v_{i, t}, \quad d_{i j}=e_{i j, t}=\frac{1}{2}\left(v_{i, j}+v_{j, i}\right) .
$$

Boundary conditions

$$
\begin{array}{lll}
\text { given acceleration: } & w_{i}=\hat{w}_{i}, \quad\left(X_{i}, t\right) \in S_{w} \times\left[t_{1}, t_{2}\right], \\
\text { given traction: } & \sigma_{i j} v_{j}=\hat{T}_{i}, \quad\left(X_{i}, t\right) \in S_{T} \times\left[t_{1}, t_{2}\right] .
\end{array}
$$

For a moored ship, at the moored point, the acceleration $\hat{W}_{i}=0$, while for a ship in motion there exists no given acceleration boundary, so that $S_{w}=0$.

Substituting Eqs. (9) and (10) into Eq. (8), we obtain the dynamic equations in its displacement form. Using the following matrix notations with the elastic modulus $E$, shear modulus $\varsigma$ and Poison's ratio $\mu$ of the material,

$$
\begin{aligned}
& \mathbf{D}=\left[\begin{array}{ccc}
\partial / \partial X_{1} & 0 & 0 \\
0 & \partial / \partial X_{2} & 0 \\
0 & 0 & \partial / \partial X_{3} \\
\partial / \partial X_{2} & \partial / \partial X_{1} & 0 \\
0 & \partial / \partial X_{3} & \partial / \partial X_{2} \\
\partial / \partial X_{3} & 0 & \partial / \partial X_{1}
\end{array}\right], \hat{\mathbf{E}}=\frac{E}{2 \varsigma(1+\mu)}\left[\begin{array}{cccccc}
1 & \gamma & \gamma & 0 & 0 & 0 \\
\gamma & 1 & \gamma & 0 & 0 & 0 \\
\gamma & \gamma & 1 & 0 & 0 & 0 \\
0 & 0 & 0 & \varsigma & 0 & 0 \\
0 & 0 & 0 & 0 & \varsigma & 0 \\
0 & 0 & 0 & 0 & 0 & \varsigma
\end{array}\right], \\
& \mathbf{u}=\left[\begin{array}{lll}
u_{1} & u_{2} & u_{3}
\end{array}\right]^{T}, \mathbf{v}=\dot{\mathbf{u}}, \quad \mathbf{w}=\ddot{\mathbf{u}}, \quad \varsigma=\frac{(1-2 \mu)}{2(1-\mu)}, \\
& \mathbf{U}=\left[\begin{array}{llll}
U_{1} & U_{2} & U_{3} & \theta_{1}
\end{array}\right]^{T}, \mathbf{V}=\dot{\mathbf{U}}, \quad \mathbf{W}=\ddot{\mathbf{U}}, \tilde{\mathbf{p}}=\left[\begin{array}{lll}
\tilde{p}_{1} & \tilde{p}_{2} & \tilde{p}_{3}
\end{array}\right]^{T},
\end{aligned}
$$




$$
\begin{aligned}
& \overline{\mathbf{v}}=\left[\begin{array}{cccccc}
v_{1} & 0 & 0 & v_{2} & 0 & v_{3} \\
0 & v_{2} & 0 & v_{1} & v_{3} & 0 \\
0 & 0 & v_{3} & 0 & v_{2} & v_{1}
\end{array}\right], \quad \mathbf{v}=\left[\begin{array}{lll}
v_{1} & v_{2} & v_{3}
\end{array}\right]^{T}, \quad \boldsymbol{\eta}=\left[\begin{array}{lll}
\eta_{1} & \eta_{2} & \eta_{3}
\end{array}\right]^{T}, \\
& \mathbf{E}=\operatorname{diag}\left(E S \quad E J_{2} \quad E J_{3} \quad G J_{1}\right), \overline{\mathbf{L}}=\operatorname{diag}\left(0, \partial / \partial Y_{1}, \partial / \partial Y_{1}, 0\right), \\
& \mathbf{L}=\operatorname{diag}\left(\partial / \partial Y_{1}, \partial^{2} / \partial Y_{1}^{2}, \partial^{2} / \partial Y_{1}^{2}, \partial / \partial Y_{1}\right), \quad \hat{\mathbf{F}}=\left[\begin{array}{lll}
\hat{F}_{1} & \hat{F}_{2} & \hat{F}_{3}
\end{array}\right]^{T} \text {, } \\
& \overline{\mathbf{M}}=\left[\begin{array}{lllllll}
\rho S & M_{p} & M_{1} & M_{2} & \cdots & M_{\hat{\jmath}} & M_{E}
\end{array}\right], \quad \tilde{\mathbf{M}}=\left[\begin{array}{llll}
\overline{\mathbf{M}}^{T} & \overline{\mathbf{J}}_{2}^{T} & \overline{\mathbf{J}}_{3}^{T} & \overline{\mathbf{J}}_{1}^{T}
\end{array}\right]^{T}, \\
& \overline{\mathbf{J}}_{i}=\left[\begin{array}{lllllll}
\rho J_{i} & I_{p i} & I_{1 i} & I_{2 i} & \cdots & I_{\hat{J} i} & I_{E i}
\end{array}\right], \quad(i=1,2,3), \quad \mathbf{J}=\left[\begin{array}{llll}
\mathbf{0}^{T} & \overline{\mathbf{J}}_{2}^{T} & \overline{\mathbf{J}}_{3}^{T} & \mathbf{0}^{T}
\end{array}\right]^{T}, \\
& \Delta=\left[\begin{array}{lllllll}
1 & \Delta\left(Y_{1}-Y_{p 1}\right) & \Delta\left(Y_{1}-Y_{11}\right) & \Delta\left(Y_{1}-Y_{21}\right) & \cdots & \Delta\left(Y_{1}-Y_{\hat{J}_{1}}\right) & \Delta\left(Y_{1}-Y_{E 1}\right)
\end{array}\right]^{T}, \\
& \mathbf{k}_{I}=\operatorname{diag}\left(k_{I 1}, k_{I 2}, k_{I 3}\right), \mathbf{k}_{E}=\operatorname{diag}\left(k_{E 1}, k_{E 2}, k_{E 3}\right), \Delta_{p i}=\Delta\left(Y_{i}-Y_{p i}^{+}\right)-\Delta\left(Y_{i}-Y_{p i}^{-}\right) \text {, } \\
& \mathbf{K}_{A}^{\Delta}=\sum_{I=1}^{\hat{I}}\left[\mathbf{k}_{I} \Delta\left(Y_{1}-Y_{I 1}^{A}\right)\right]+\mathbf{k}_{E} \Delta\left(Y_{1}-Y_{E 1}^{A}\right), \mathbf{K}_{B}^{\Delta}=\left\{\sum_{I=1}^{\hat{I}}\left[\mathbf{k}_{I} \Delta\left(X_{k}-X_{I k}^{B}\right)\right]+\mathbf{k}_{E} \Delta\left(X_{k}-X_{E k}^{B}\right)\right\}, \\
& \mathbf{F}_{s f}^{\Delta}=\left[\begin{array}{ll}
\left(\Delta_{p} \mathbf{A}_{p}\right)^{T} & 0
\end{array}\right]^{T}, \quad \mathbf{A}_{p}=\left[A_{p 1} A_{p 2} A_{p 3}\right]^{T}, \quad k_{E}^{\Delta}=k_{E} \Delta_{B}, \quad \Delta_{p}=\operatorname{diag}\left(\begin{array}{ccc}
\Delta_{p 1} & \Delta_{p 2} & \Delta_{p 3}
\end{array}\right) \\
& \overline{\mathbf{K}}_{B}^{\Delta}=\left[\begin{array}{cc}
\mathbf{K}_{B}^{\Delta} & \mathbf{0} \\
\mathbf{0} & 0
\end{array}\right], \overline{\mathbf{K}}_{B E}^{\Delta}=\left[\begin{array}{cc}
\mathbf{K}_{B}^{\Delta} & \mathbf{0} \\
\mathbf{0} & -k_{E}^{\Delta}
\end{array}\right], \overline{\mathbf{K}}_{A}^{\Delta}=\left[\begin{array}{cc}
\mathbf{K}_{A}^{\Delta} & 0 \\
0 & 0
\end{array}\right], \overline{\mathbf{K}}_{A E}^{\Delta}=\left[\begin{array}{cc}
\mathbf{K}_{A}^{\Delta} & 0 \\
0 & -k_{E}^{\Delta}
\end{array}\right], \overline{\boldsymbol{\beta}}=\left[\begin{array}{c}
\boldsymbol{\beta} \\
\boldsymbol{\beta}_{3}
\end{array}\right],
\end{aligned}
$$

We can write this equation in a matrix form

$$
\mathbf{D}^{T}(\hat{\mathbf{E} D u})+\hat{\mathbf{F}}-\overline{\boldsymbol{\beta}}^{T} \overline{\mathbf{K}}_{B E}^{\Delta} \mathbf{U}+\overline{\boldsymbol{\beta}}^{T} \overline{\mathbf{K}}_{B}^{\Delta} \overline{\boldsymbol{\beta}} \mathbf{u}=\rho_{s} \mathbf{W}
$$

The corresponding boundary conditions (12) in a matrix form are as follows

$$
\text { given acceleration: } \quad \mathbf{w}=\hat{\mathbf{w}},
$$

given traction:

$$
\overline{\mathbf{v}} \hat{\mathbf{E} D u}=\hat{\mathbf{T}} \text {. }
$$

\subsection{Fluid domain}

The water motion is described by a dynamic pressure relative to the stationary travel state where this dynamic pressure vanishing. The wave equation and boundary conditions for water domain are given as follows (Xing, 1991).

Dynamic equation

$$
p_{, t t}=c^{2} p_{, i i}
$$$$
\left(x_{i}, t\right) \in \Omega_{f} \times\left(t_{1}, t_{2}\right) .
$$

Boundary conditions 

transmitted to infinity due to practical damping, or to Sommerfeld radiation condition implying waves transmitting to infinity without any reflections (Xing, 2007; Xing, 2008). This paper adopts the zero disturbance condition at a sufficiently far boundary from the ship. An incident wave excitation can be modelled by equation (16-2) where the dynamic pressure is given, or by equation (16-3) which gives the acceleration of the incident wave.

2.4 Water-hull interaction interface

where $\nabla=\left[\begin{array}{lll}\partial / \partial x_{1} & \partial / \partial x_{2} & \partial / \partial x_{3}\end{array}\right]^{T}$

\subsection{Propulsion unit}

The motion of the propulsion unit is investigated in the shaft system $\hat{o}-Y_{1} Y_{2} Y_{3}$, which consists of an axial extension / compression displacement $U_{1}\left(Y_{j}, t\right)$, two bending displacements $U_{2}\left(Y_{j}, t\right)$ and $U_{3}\left(Y_{j}, t\right)$ as well as a rotation about $\hat{o}-Y_{1}$ axis. We neglected the couplings between these four types of motions. Based on the classical theory of road, shaft and beam, using the second Newton's law, we can derive the dynamic equations describing the dynamics of the propulsion unit as follows.

Axial extension $U_{1}\left(Y_{j}, t\right)$ and Torsion $\theta_{1}\left(Y_{1}, t\right)$

$$
\begin{gathered}
\frac{\partial}{\partial Y_{1}}\left(E S \frac{\partial U_{1}}{\partial Y_{1}}\right)+\tilde{f}_{p 1}^{s} \Delta\left(Y_{1}-Y_{p 1}\right)+\sum_{I=1}^{\hat{I}} \tilde{f}_{I 1}^{A s} \Delta\left(Y_{1}-Y_{I 1}^{A}\right)+\tilde{f}_{E 1}^{A s} \Delta\left(Y_{1}-Y_{E 1}^{A}\right)=\overline{\mathbf{M}} \Delta \ddot{U}_{1}, \\
\frac{\partial}{\partial Y_{1}}\left(G J_{1} \frac{\partial \theta_{1}}{\partial Y_{1}}\right)+\tilde{m}_{E}^{s}=\overline{\mathbf{J}}_{1} \Delta \ddot{\theta}_{1},
\end{gathered}
$$

Bending displacements $U_{2}\left(Y_{j}, t\right)$ and $U_{3}\left(Y_{j}, t\right)$ 


$$
\frac{\partial^{2}}{\partial Y_{1}^{2}}\left(E J_{2} \frac{\partial^{2} U_{2}}{\partial Y_{1}^{2}}\right)+\tilde{f}_{p 2}^{s} \Delta\left(Y_{1}-Y_{p 1}\right)+\sum_{I=1}^{\hat{I}} \tilde{f}_{I 2}^{A s} \Delta\left(Y_{1}-Y_{I 1}^{A}\right)
$$

$$
\begin{gathered}
+\tilde{f}_{E 2}^{A s} \Delta\left(Y_{1}-Y_{E 1}^{A}\right)=-\overline{\mathbf{M}} \Delta \ddot{U}_{2}+\overline{\mathbf{J}}_{2} \Delta \frac{\partial^{2} \ddot{U}_{2}}{\partial Y_{1}^{2}} . \\
\frac{\partial^{2}}{\partial Y_{1}^{2}}\left(E J_{3} \frac{\partial^{2} U_{3}}{\partial Y_{1}^{2}}\right)+\tilde{f}_{p 3}^{s} \Delta\left(Y_{1}-Y_{p 1}\right)+\sum_{I=1}^{\hat{I}} \tilde{f}_{I 3}^{A s} \Delta\left(Y_{1}-Y_{I 1}^{A}\right) \\
+\tilde{f}_{E 3}^{A s} \Delta\left(Y_{1}-Y_{E 1}^{A}\right)=-\overline{\mathbf{M}} \Delta \ddot{U}_{3}+\overline{\mathbf{J}}_{3} \Delta \frac{\partial^{2} \ddot{U}_{3}}{\partial Y_{1}^{2}} .
\end{gathered}
$$

Eqs. (18)-(21) are rewritten in a matrix form

$$
-\boldsymbol{L}(\boldsymbol{E L} \boldsymbol{U})+\overline{\boldsymbol{K}}_{A}^{\Delta} \boldsymbol{U}-\overline{\boldsymbol{K}}_{A E}^{\Delta} \overline{\boldsymbol{\beta}} \boldsymbol{u}+\tilde{\boldsymbol{f}}_{p}=\left[\operatorname{diag}(\tilde{\boldsymbol{M}} \boldsymbol{\Delta})-\operatorname{diag}(\boldsymbol{J} \boldsymbol{\Delta}) \overline{\boldsymbol{L}}^{2}\right] \ddot{\boldsymbol{U}}
$$

In this equation, the third and fourth terms represent the interactions of propulsion unit with the hull and the water, respectively.

\subsection{Propeller shaft-water interaction interface}

Assume that $\bar{\Gamma}_{P}$ denotes the interaction wet interface between the shaft and water, in which the points $Y_{i}=Y_{p i}, x_{i}=x_{p i}$ are located, the averaged approximately coupling conditions may be represented as

$$
\begin{array}{lrr}
\text { kinematic: } & \ddot{\mathbf{U}}^{T} \mathbf{F}_{s f}^{\Delta}=-\int_{\bar{\Gamma}_{p}} \boldsymbol{\eta}^{T} \nabla p / \rho_{f} d \Gamma, & Y_{i}=Y_{p i}, x_{i}=x_{p i}, \\
\text { equilibrium: } & \tilde{\mathbf{f}}_{p}^{s}=-\tilde{\mathbf{f}}_{p}=-\mathbf{F}_{s f}^{\Delta} p, & Y_{i}=Y_{p i}, \quad x_{i}=x_{p i} .
\end{array}
$$

\section{Variational formulation}

In order to construct a numerical model for the coupling system, we need to establish a variational formulation of which the stationary conditions cover all of the governing equations presented above. Based on the generalised variational principles developed by Xing et al (Xing, 1995; Xing, 1996), we can construct the following functional, 


$$
\begin{aligned}
& H_{s f}[p, \mathbf{W}, \mathbf{W}]=\int_{t_{1}}^{t_{2}}\left\{\int _ { Y _ { p 1 } } ^ { Y _ { E _ { 1 } } } \left[\frac{1}{2} \mathbf{W}^{T} \operatorname{diag}(\tilde{\mathbf{M}} \boldsymbol{\Delta}) \mathbf{W}+\frac{1}{2} \mathbf{W}_{, 1}^{T} \operatorname{diag}(\mathbf{J} \Delta) \mathbf{W}_{, 1}-\frac{1}{2}\left(\dot{\mathbf{U}}^{T} \overline{\mathbf{L}}^{T}\right) \mathbf{E} \overline{\mathbf{L}} \dot{\mathbf{U}}\right.\right. \\
& \left.-\frac{1}{2}\left[\begin{array}{ll}
\dot{\mathbf{U}}^{T} & \dot{\mathbf{u}}^{T} \overline{\boldsymbol{\beta}}^{T}
\end{array}\left[\begin{array}{cc}
\overline{\mathbf{K}}_{A}^{\Delta} & -\overline{\mathbf{K}}_{A E}^{\Delta} \\
-\overline{\mathbf{K}}_{B E}^{\Delta} & \overline{\mathbf{K}}_{B}^{\Delta}
\end{array}\right]\left[\begin{array}{c}
\dot{\mathbf{U}} \\
\overline{\boldsymbol{\beta}} \dot{\mathbf{u}}
\end{array}\right]-\mathbf{W}^{T} \mathbf{F}_{s f}^{\Delta} p\right] d L\right\} d t \\
& +\int_{t_{1}}^{t_{2}}\left\{\int_{\Omega_{s}}\left[\frac{1}{2} \rho_{s} \mathbf{w}^{T} \mathbf{w}-\frac{1}{2}\left(\dot{\mathbf{u}}^{T} \mathbf{D}^{T}\right) \hat{\mathbf{E}} \mathbf{D} \dot{\mathbf{u}}-\mathbf{w}^{T} \hat{\mathbf{F}}\right] d \Omega_{s}-\int_{S_{T}} \mathbf{w}^{T} \hat{\mathbf{T}} d S\right\} d t \\
& +\int_{t_{1}}^{t_{2}}\left\{\int_{\Omega_{f}}\left[\frac{1}{2 \rho_{f} c^{2}} p_{, t} p_{, t}-\frac{1}{2 \rho_{f}} p \nabla^{T} \nabla p\right] d \Omega_{f}+\int_{\Gamma_{f}} \frac{\eta_{3}}{2 \rho_{f} g} p_{, t} p_{, t} d \Gamma-\int_{\Gamma_{w}} p \hat{\mathbf{w}}^{T} \boldsymbol{\eta} d \Gamma\right. \\
& \left.-\int_{\Sigma}\left(p \mathbf{w}^{T} \boldsymbol{\eta}+\frac{1}{2} \rho_{f} g \dot{u}_{3}^{2}\right) d \Gamma\right\} d t
\end{aligned}
$$

In this functional, the acceleration in solids and the pressure in fluids are taken as the variables. The functional is subject to the constraints given in Eqs. (9), (11), (14-2), (16-2) as well as the imposed variation constraints $\delta v_{i}=0=\delta p$ at the two time terminals $t_{1}$ and $t_{2}$. The stationary conditions of the functional given in Eq. (24) are described in Eqs. (14), (15), (16-1,3), (17), (22) and (23).

\section{Substructures and their mode functions}

To establish a numerical model to investigate the dynamics of the integrated coupling system governed by the functional (24), we need to find some Ritz functions to represent the motion of the system. For this purpose, we divide the integrated system into two solid substructures (Xing, 1986; Xing, 1986; Craig et al, 1986; Xing, 1983) (a hull and a shaft) and a fluid domain (Xing, 1996). We derive the natural frequencies and mode functions of each substructure / domain as follows. These mode functions will be chosen as Ritz functions to span a subspace in which the motion of substructure / domain is described.

4.1 Hull substructure and its mode functions

The free-free dry hull with no the shaft system is considered as the hull substructure of which the equation for the natural vibrations with no any external forces are derived from equation (14), i.e.

$$
\begin{aligned}
& \mathbf{D}^{T}(\hat{\mathbf{E} D u})=\rho_{s} \ddot{\mathbf{u}}, \\
& \overline{\mathbf{v}} \hat{\mathbf{E}} \mathbf{D u}=0 .
\end{aligned}
$$




$$
\mathbf{u}=\boldsymbol{\varphi}_{I} e^{\mathrm{j} \omega_{I} \mathrm{t}}, \quad \ddot{\mathbf{u}}=-\omega^{2} \boldsymbol{\varphi}_{I} e^{\mathrm{j} \omega_{I} \mathrm{t}}
$$

267 from which, when substituted into equation (25), it follows

$$
\mathbf{D}^{T}\left(\hat{\mathbf{E} D} \boldsymbol{\varphi}_{I}\right)=-\omega_{I}^{2} \rho_{s} \boldsymbol{\varphi}_{I},
$$

$$
\overline{\mathbf{v}} \hat{\mathbf{E D}} \varphi_{I}=0 \text {. }
$$

269

Pre-multiplying equation (27) by $\varphi_{I}^{T}$ and then integrating it over the volume of the hull as well as using Green theorem, we obtain

$$
\begin{gathered}
\int_{\Omega_{S}} \boldsymbol{\varphi}_{I}^{T} \mathbf{D}^{T}\left(\hat{\mathbf{E}} \mathbf{D} \boldsymbol{\varphi}_{I}\right) d \Omega_{S}=\int_{S} \overline{\mathbf{v}} \hat{\mathbf{E}} \mathbf{D} \boldsymbol{\varphi}_{I} d S-\int_{\Omega_{S}}\left(\boldsymbol{\varphi}_{I}^{T} \mathbf{D}^{T}\right) \hat{\mathbf{E}} \mathbf{D} \boldsymbol{\varphi}_{I} d \Omega_{S}=-k_{I} \\
=-\int_{\Omega_{S}} \omega_{I}^{2} \boldsymbol{\varphi}_{I}^{T} \rho_{S} \boldsymbol{\varphi}_{I} d \Omega_{S}=-\omega_{I}^{2} m_{I}, \\
k_{I}=\int_{\Omega_{S}} \boldsymbol{\varphi}_{I}^{T} \mathbf{D}^{T} \hat{\mathbf{E}} \mathbf{D} \boldsymbol{\varphi}_{I} d \Omega_{S}, \quad m_{I}=\int_{\Omega_{S}} \omega_{I}^{2} \boldsymbol{\varphi}_{I}^{T} \rho_{s} \boldsymbol{\varphi}_{I} d \Omega_{S}, \quad \omega_{I}^{2}=k_{I} / m_{I} .
\end{gathered}
$$

Here, $k_{I}$ and $m_{I}$ are called the generalised stiffness and mass of the I-th mode of the hull. For any two modes $\boldsymbol{\varphi}_{I}$ and $\boldsymbol{\varphi}_{J}$ with difference frequencies, the following orthogonal relationships are valid

$$
\int_{\Omega_{S}} \boldsymbol{\varphi}_{I}^{T} \mathbf{D}^{T} \hat{\mathbf{E}} \mathbf{D} \boldsymbol{\varphi}_{J} d \Omega_{S}=\left\{\begin{array}{cc}
0, & I \neq J \\
k_{I}, & I=J
\end{array}, \quad \mathbf{k}=\operatorname{diag}\left(k_{I}\right),\right.
$$

$$
\int_{\Omega_{S}} \boldsymbol{\varphi}_{I}^{T} \rho_{s} \boldsymbol{\varphi}_{J} d \Omega_{S}=\left\{\begin{array}{cc}
0, & I \neq J \\
m_{I}, & I=J
\end{array}, \quad \mathbf{m}=\operatorname{diag}\left(m_{I}\right) .\right.
$$

Generally, the first $\mathrm{n}$ natural frequencies and modes can be solved by finite element method and corresponding computer code. Introducing the eigen-matrices of the hull

$$
\lambda=\operatorname{diag}\left(\omega_{1}, \omega_{2}, \cdots, \omega_{\mathrm{n}}\right), \quad \boldsymbol{\varphi}=\left[\begin{array}{llll}
\boldsymbol{\varphi}_{1} & \boldsymbol{\varphi}_{2} & \cdots & \boldsymbol{\varphi}_{3}
\end{array}\right],
$$

which is used as a generalised coordinate frame to describe the motion of the hull. We represent the displacement of the hull in the form

$$
\mathbf{u}=\boldsymbol{\varphi q}, \quad \dot{\mathbf{u}}=\boldsymbol{\varphi} \dot{\mathbf{q}}, \quad \ddot{\mathbf{u}}=\boldsymbol{\varphi} \ddot{\mathbf{q}}, \quad \mathbf{q}=\left[\begin{array}{llll}
q_{1} & q_{2} & \cdots & q_{n}
\end{array}\right]^{T},
$$

where $\mathbf{q}$ called as the generalised coordinate vector that is a time function.

4.2 Propulsion unit substructure and its mode functions

The free-free propulsion shaft with its attached disks, propeller and etc is considered as a substructure of which the equations for the natural vibrations with no any external forces are derived from Eq. (22), 


$$
-\mathbf{L}(\mathbf{E L U})=\left[\operatorname{diag}(\tilde{\mathbf{M}} \boldsymbol{\Delta})-\operatorname{diag}(\mathbf{J} \boldsymbol{\Delta}) \overline{\mathbf{L}}^{2}\right] \ddot{\mathbf{U}},
$$

$$
\mathbf{E L U}=0, \quad \overline{\mathbf{L}}(\mathbf{E L U})=0, \quad Y_{1}=Y_{p 1}, Y_{E 1} \text {. }
$$

Also, this is an eigenvalue problem from which the I-th natural frequencies $\Omega_{I}$ and the corresponding

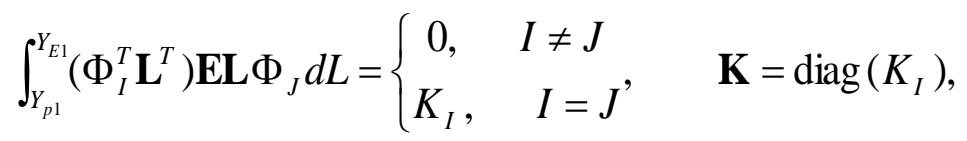

$$
\int_{Y_{p 1}}^{Y_{E 1}} \Phi_{I}^{T}\left[\operatorname{diag}(\tilde{\mathbf{M}} \boldsymbol{\Delta})+\overline{\mathbf{L}}^{T} \operatorname{diag}(\mathbf{J} \boldsymbol{\Delta}) \overline{\mathbf{L}}\right] \Phi_{J} d L=\left\{\begin{array}{cc}
0, & I \neq J \\
M_{I}, & I=J
\end{array}, \quad \mathbf{M}=\operatorname{diag}\left(M_{I}\right) .\right.
$$
unit

mode function $\Phi_{I}$ can be obtained. We assume that

$$
\mathbf{U}=\Phi_{I} e^{\mathrm{j} \Omega_{I} \mathrm{t}}, \quad \ddot{\mathbf{U}}=-\Omega_{I}^{2} \Phi_{I} e^{\mathrm{j} \Omega_{I} \mathrm{t}},
$$

from which, when substituted into equation (31), it follows

$$
\begin{aligned}
& -\mathbf{L}\left(\mathbf{E L} \Phi_{I}\right)=-\Omega_{I}^{2}[\operatorname{diag}(\tilde{\mathbf{M}} \mathbf{\Delta})-\operatorname{diag}(\mathbf{J} \mathbf{\Delta}) \mathbf{L}] \Phi_{I}, \\
& \mathbf{E} \mathbf{L} \Phi_{I}=0, \quad \overline{\mathbf{L}}\left(\mathbf{E} \mathbf{L} \Phi_{I}\right)=0, \quad Y_{1}=Y_{p 1}, Y_{E 1} .
\end{aligned}
$$

Pre-multiplying equation (33) by $\Phi_{I}^{T}$ and then integrating it over the length of the shaft as well as integrating it by parts we obtain

$$
K_{I}=\Omega_{I}^{2} M_{I}, \quad K_{I}=\int_{Y_{p 1}}^{Y_{E 1}}\left(\Phi_{I}^{T} \overline{\mathbf{L}}^{T}\right) \mathbf{E L} \Phi_{I} d L, \quad M_{I}=\int_{Y_{p 1}}^{Y_{E 1}} \Phi_{I}^{T}\left[\operatorname{diag}(\tilde{\mathbf{M}} \boldsymbol{\Delta})+\overline{\mathbf{L}}^{T} \operatorname{diag}(\mathbf{J} \boldsymbol{\Delta}) \overline{\mathbf{L}}\right] \Phi_{I} d L
$$

Here, $K_{I}$ and $M_{I}$ are called the generalised stiffness and mass of the I-th mode of the shaft. For any two modes $\Phi_{I}$ and $\Phi_{J}$ with difference frequencies, the following orthogonal relationships are valid

$$
\boldsymbol{\Lambda}=\operatorname{diag}\left(\Omega_{1}, \Omega_{2}, \cdots, \Omega_{N}\right), \quad \boldsymbol{\Phi}=\left[\begin{array}{llll}
\Phi_{1} & \Phi_{2} & \cdots & \Phi_{N}
\end{array}\right]
$$

which is used as a generalised coordinate frame to describe the motion of the shaft. We represent the displacement of the shaft in the form

$$
\mathbf{U}=\boldsymbol{\Phi} \mathbf{Q}, \quad \dot{\mathbf{U}}=\mathbf{\Phi} \dot{\mathbf{Q}}, \quad \ddot{\mathbf{U}}=\mathbf{\Phi} \ddot{\mathbf{Q}}, \quad \mathbf{Q}=\left[\begin{array}{llll}
Q_{1} & Q_{2} & \cdots & Q_{N}
\end{array}\right]^{T},
$$

where $\mathbf{Q}$ called as the generalised coordinate vector that is a time function. 
308

309

319

$$
\left.\left(\rho_{f}\right)^{-1} \int_{\Omega_{f}} \Psi_{I, i}^{T} \Psi_{J, i}\right) d \Omega_{f}=\left\{\begin{array}{cc}
0, & I \neq J \\
\tilde{k}_{I}, & I=J
\end{array}, \quad \tilde{\mathbf{k}}=\operatorname{diag}\left(\tilde{k}_{I}\right),\right.
$$

$$
\left(\rho_{f} c^{2}\right)^{-1} \int_{\Omega_{f}} \Psi_{I}^{T} \Psi_{I} d \Omega_{f}+\left(\rho_{f} g\right)^{-1} \int_{\Gamma_{f}} \Psi_{I}^{T} \Psi_{I} d \Gamma=\left\{\begin{array}{cc}
0, & I \neq J \\
\tilde{m}_{I}, & I=J
\end{array}, \quad \tilde{\mathbf{m}}=\operatorname{diag}\left(\tilde{m}_{I}\right) .\right.
$$

4.3 Water domain and its mode functions

The water is considered as a subdomain of which the natural vibration is governed by the following equations derived from Eq. (15) and Eq. (16) by fixing its boundaries except free surface, that is

$$
\begin{aligned}
& p_{, t t}=c^{2} p_{, i i}, \quad\left(x_{i}, t\right) \in \Omega_{f} \times\left(t_{1}, t_{2}\right), \\
& p_{, i} \eta_{i}=-p_{, t t} / g, \quad\left(x_{i}, t\right) \in \Gamma_{f} \times\left[t_{1}, t_{2}\right], \\
& p_{, i} \eta_{i}=0, \quad\left(x_{i}, t\right) \in \Gamma_{w} \cup \sum \times\left[t_{1}, t_{2}\right] .
\end{aligned}
$$

Here, we consider the incident wave is given by a boundary acceleration so that $\Gamma_{p}=0$. This set of equations constructs a fluid pressure eigenvalue problem, and to obtain its solution we assume that

$$
p=\Psi_{I} e^{\mathrm{j} \tilde{\omega}_{I} \mathrm{t}}, \quad p_{, t t}=-\tilde{\omega}_{I}^{2} \Psi_{I} e^{\mathrm{j} \tilde{\omega}_{I} \mathrm{t}},
$$

from which, when substituted into Eq. (38), it follows

$$
\begin{aligned}
& -\tilde{\omega}_{I}^{2} \Psi_{I}=c^{2} \Psi_{I, i i}, \quad\left(x_{i}, t\right) \in \Omega_{f} \times\left(t_{1}, t_{2}\right), \\
& \Psi_{I, i} \eta_{i}=\tilde{\omega}_{I}^{2} \Psi_{I} / g, \quad\left(x_{i}, t\right) \in \Gamma_{f} \times\left[t_{1}, t_{2}\right], \\
& \Psi_{I, i} \eta_{i}=0, \quad\left(x_{i}, t\right) \in \Gamma_{w} \cup \Sigma \times\left[t_{1}, t_{2}\right] .
\end{aligned}
$$

Pre-multiplying Eq. (40) by $\Psi_{I}^{T}\left(\rho_{f} c^{2}\right)^{-1}$ and then integrating it over the water domain and using Green theorem, we obtain

$$
\begin{aligned}
& \left.\tilde{\omega}_{I}^{2}\left[\left(\rho_{f} c^{2}\right)^{-1} \int_{\Omega_{f}} \Psi_{I}^{T} \Psi_{I} d \Omega_{f}+\left(\rho_{f}\right)^{-1} \int_{\Gamma_{f}} \Psi_{I}^{T} \Psi_{I} / g d \Gamma\right]=\left(\rho_{f}\right)^{-1} \int_{\Omega_{f}} \Psi_{I, i}^{T} \Psi_{I, i}\right) d \Omega_{f}, \\
& \left.\tilde{k}_{I}=\left(\rho_{f}\right)^{-1} \int_{\Omega_{f}} \Psi_{I, i}^{T} \Psi_{I, i}\right) d \Omega_{f}, \quad \tilde{\omega}_{I}^{2}=\tilde{k}_{I} / \tilde{m}_{I}, \\
& \tilde{m}_{I}=\left(\rho_{f} c^{2}\right)^{-1} \int_{\Omega_{f}} \Psi_{I}^{T} \Psi_{I} d \Omega_{f}+\left(\rho_{f} g\right)^{-1} \int_{\Gamma_{f}} \Psi_{I}^{T} \Psi_{I} d \Gamma,
\end{aligned}
$$

\footnotetext{
Introducing the eigen-matrices of the first $\tilde{n}$ natural frequencies and corresponding modes fort the
} 
water

$$
\tilde{\lambda}=\operatorname{diag}\left(\tilde{\omega}_{1}, \tilde{\omega}_{2}, \cdots, \tilde{\omega}_{\tilde{n}}\right), \quad \boldsymbol{\Psi}=\left[\begin{array}{llll}
\Psi_{1} & \Psi_{2} & \ldots & \Psi_{\tilde{n}}
\end{array}\right]
$$

323

324

which is used as a generalised coordinate frame to describe the motion of the water. We represent the dynamics pressure of the water in the form

$$
p=\boldsymbol{\Psi} \tilde{\mathbf{q}}, \quad p_{, t}=\boldsymbol{\Psi} \dot{\tilde{\mathbf{q}}}, \quad p_{, t t}=\boldsymbol{\Psi} \ddot{\tilde{\mathbf{q}}}, \quad \tilde{\mathbf{q}}=\left[\begin{array}{llll}
\tilde{q}_{1} & \tilde{q}_{2} & \cdots & \tilde{q}_{\tilde{n}}
\end{array}\right]^{T},
$$

where $\tilde{\mathbf{q}}$ is a time function and called as the generalised coordinate vector of the fluid

For practical complex ship structures and fluid domains, the mode functions can be derived using finite element methods (Bathe, 1983; Zienkiewicz, 1991). The developed computer code for fluidstructure dynamic analysis can provide a mean to complete these calculations (Xing, 1995; Xing, 1995).

\section{Mode equation of the integrated coupling system}

Substituting Eqs. (30), (37) and (44) into the functional (24), we obtain that

$H_{s f}[\widetilde{\mathbf{q}}, \mathbf{q}, \mathbf{Q}]=\int_{t_{1}}^{t_{2}}\left\{\int_{Y_{p 1}}^{Y_{E 1}}\left\{\frac{1}{2} \ddot{\mathbf{Q}}^{T} \boldsymbol{\Phi}^{T}\left[\operatorname{diag}(\tilde{\mathbf{M}} \Delta)+\overline{\mathbf{L}}^{T} \operatorname{diag}(\mathbf{J} \boldsymbol{\Delta}) \overline{\mathbf{L}}\right] \boldsymbol{\Phi} \ddot{\mathbf{Q}}-\frac{1}{2} \dot{\mathbf{Q}}^{T} \boldsymbol{\Phi}^{T} \overline{\mathbf{L}}^{T} \mathbf{E} \overline{\mathbf{L}} \boldsymbol{\Phi} \dot{\mathbf{Q}}\right.\right.$

$\left.-\frac{1}{2}\left[\begin{array}{cc}\dot{\mathbf{Q}}^{T} & \dot{\mathbf{q}}^{T}\end{array}\left[\begin{array}{cc}\boldsymbol{\Phi}^{T} & \mathbf{0} \\ \mathbf{0} & \boldsymbol{\varphi}^{T} \overline{\boldsymbol{\beta}}^{T}\end{array}\right]\left[\begin{array}{cc}\overline{\mathbf{K}}_{A}^{\Delta} & -\overline{\mathbf{K}}_{A E}^{\Delta} \\ -\overline{\mathbf{K}}_{B E}^{\Delta} & \overline{\mathbf{K}}_{B}^{\Delta}\end{array}\right]\left[\begin{array}{cc}\mathbf{\Phi} & \mathbf{0} \\ \mathbf{0} & \overline{\boldsymbol{\beta}} \boldsymbol{\varphi}\end{array}\right]\left[\begin{array}{c}\dot{\mathbf{Q}} \\ \dot{\mathbf{q}}\end{array}\right]-\ddot{\mathbf{Q}}^{T} \mathbf{\Phi}^{T} \mathbf{F}_{s f}^{\Delta} \boldsymbol{\Psi} \tilde{\mathbf{q}}\right\} d L\right\} d t$

$+\int_{t_{1}}^{t_{2}}\left\{\int_{\Omega_{s}}\left[\frac{1}{2} \ddot{\mathbf{q}}^{T} \boldsymbol{\varphi}^{T} \rho_{s} \boldsymbol{\varphi} \ddot{\mathbf{q}}-\frac{1}{2} \dot{\mathbf{q}}^{T}\left(\boldsymbol{\varphi}^{T} \mathbf{D}^{T} \hat{\mathbf{E}} \mathbf{D} \boldsymbol{\varphi}\right) \dot{\mathbf{q}}-\ddot{\mathbf{q}}^{T} \boldsymbol{\varphi}^{T} \hat{\mathbf{F}}\right] d \Omega_{s}-\int_{S_{T}} \ddot{\mathbf{q}}^{T} \boldsymbol{\varphi}^{T} \hat{\mathbf{T}} d S\right\} d t$

$+\int_{t_{1}}^{t_{2}}\left\{\int_{\Omega_{f}}\left[\frac{1}{2 \rho_{f} c^{2}} \dot{\tilde{\mathbf{q}}}^{T} \boldsymbol{\Psi}^{T} \boldsymbol{\Psi} \dot{\tilde{\mathbf{q}}}-\frac{1}{2 \rho_{f}} \widetilde{\mathbf{q}}^{T} \boldsymbol{\Psi}_{, i}^{T} \boldsymbol{\Psi}_{, i} \tilde{\mathbf{q}}\right] d \Omega_{f}\right\} d t$

$+\int_{t_{1}}^{t_{2}}\left\{\int_{\Gamma_{f}} \frac{\eta_{3}}{2 \rho_{f} g} \dot{\tilde{\mathbf{q}}}{ }^{T} \boldsymbol{\Psi}^{T} \boldsymbol{\Psi} \dot{\tilde{\mathbf{q}}} d \Gamma-\int_{\Gamma_{w}} \tilde{\mathbf{q}}^{T} \boldsymbol{\Psi}^{T} \hat{\mathbf{w}}^{T} \boldsymbol{\eta} d \Gamma-\int_{\Sigma}\left(\tilde{\mathbf{q}}^{T} \boldsymbol{\Psi}^{T} \boldsymbol{\eta}^{T} \boldsymbol{\varphi} \ddot{\mathbf{q}}+\frac{1}{2} \dot{\mathbf{q}}^{T} \boldsymbol{\varphi}_{3}^{T} \rho_{f} g \boldsymbol{\varphi}_{3} \dot{\mathbf{q}}\right) d \Gamma\right\} d t$,

into which, when Eqs. (28), (35) and (42) is substituted, it follows

$$
\begin{aligned}
& H_{s f}[\widetilde{\mathbf{q}}, \mathbf{q}, \mathbf{Q}]=\int_{t_{1}}^{t_{2}}\left\{\frac{1}{2} \ddot{\mathbf{Q}}^{T} \mathbf{M} \ddot{\mathbf{Q}}-\frac{1}{2} \dot{\mathbf{Q}}^{T} \mathbf{K} \dot{\mathbf{Q}}-\frac{1}{2}\left[\dot{\mathbf{Q}}^{T} \quad \dot{\mathbf{q}}^{T}\left[\begin{array}{ll}
\mathbf{K}_{s s} & \mathbf{K}_{s h} \\
\mathbf{K}_{h s} & \mathbf{K}_{h h}
\end{array}\right]\left[\begin{array}{c}
\dot{\mathbf{Q}} \\
\dot{\mathbf{q}}
\end{array}\right]-\ddot{\mathbf{Q}}^{T} \mathbf{K}{ }_{s w} \tilde{\mathbf{q}}\right\} d t\right. \\
& +\int_{t_{1}}^{t_{2}}\left(\frac{1}{2} \ddot{\mathbf{q}}^{T} \mathbf{m} \ddot{\mathbf{q}}-\frac{1}{2} \dot{\mathbf{q}}^{T}(\mathbf{k}+\mathbf{k} g) \dot{\mathbf{q}}-\ddot{\mathbf{q}}^{T} \hat{\mathbf{F}}_{h}\right) d t+\int_{t_{1}}^{t_{2}}\left(\frac{1}{2} \dot{\tilde{\mathbf{q}}}^{T} \tilde{\mathbf{m}} \dot{\tilde{\mathbf{q}}}-\frac{1}{2} \widetilde{\mathbf{q}}^{T} \tilde{\mathbf{k}} \tilde{\mathbf{q}}\right) d t \\
& -\int_{t_{1}}^{t_{2}}\left(\tilde{\mathbf{q}}^{T} \hat{\mathbf{F}}_{w}+\tilde{\mathbf{q}}^{T} \mathbf{K}_{h w}^{T} \ddot{\mathbf{q}}\right) d t,
\end{aligned}
$$

where 


$$
\begin{aligned}
& {\left[\begin{array}{ll}
\mathbf{K}_{s s} & \mathbf{K}_{s h} \\
\mathbf{K}_{h s} & \mathbf{K}_{h h}
\end{array}\right]=\int_{Y_{p 1}}^{Y_{E}}\left[\begin{array}{cc}
\boldsymbol{\Phi}^{T} & \mathbf{0} \\
\mathbf{0} & \boldsymbol{\varphi}^{T} \overline{\boldsymbol{\beta}}^{T}
\end{array}\right]\left[\begin{array}{cc}
\overline{\mathbf{K}}_{A}^{\Delta} & -\overline{\mathbf{K}}_{A E}^{\Delta} \\
-\overline{\mathbf{K}}_{B E}^{\Delta} & \overline{\mathbf{K}}_{B}^{\Delta}
\end{array}\right]\left[\begin{array}{cc}
\boldsymbol{\Phi} & \mathbf{0} \\
\mathbf{0} & \overline{\boldsymbol{\beta}} \boldsymbol{\varphi}
\end{array}\right] d L,} \\
& \mathbf{K}_{s w}=\int_{Y_{p 1}}^{Y_{E 1}} \boldsymbol{\Phi}^{T} \mathbf{F}_{s f}^{\Delta} \boldsymbol{\Psi} d L, \quad \mathbf{K}_{h w}=\int_{\Sigma} \boldsymbol{\varphi}^{T} \boldsymbol{\eta} \boldsymbol{\Psi} d \Gamma,
\end{aligned}
$$

$$
\begin{aligned}
& \hat{\mathbf{F}}_{h}=\int_{\Omega_{s}} \boldsymbol{\varphi}^{T} \hat{\mathbf{F}} d \Omega_{s}+\int_{S_{T}} \boldsymbol{\varphi}^{T} \hat{\mathbf{T}} d S, \quad \hat{\mathbf{F}}_{w}=\int_{\Gamma_{w}} \boldsymbol{\Psi}^{T} \hat{\mathbf{w}}^{T} \boldsymbol{\eta} d \Gamma, \\
& \mathbf{k}_{g}=\int_{\Sigma} \boldsymbol{\varphi}_{3}^{T} \rho_{f} g \boldsymbol{\varphi}_{3} d \Gamma .
\end{aligned}
$$

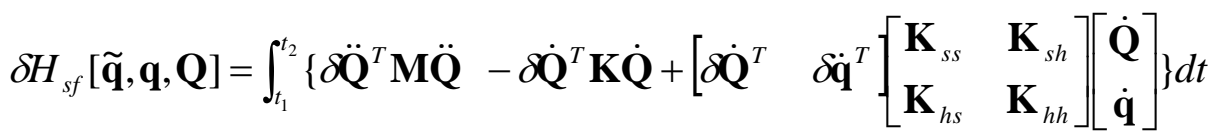

$$
\begin{aligned}
& +\int_{t_{1}}^{t_{2}}\left(\delta \ddot{\mathbf{q}}^{T} \mathbf{m} \ddot{\mathbf{q}}-\delta \dot{\mathbf{q}}^{T}\left(\mathbf{k}+\mathbf{k}_{g}\right) \dot{\mathbf{q}}-\delta \dot{\mathbf{q}}^{T} \hat{\mathbf{F}}_{h}\right) d t+\int_{t_{1}}^{t_{2}}\left(\dot{\tilde{\mathbf{q}}}^{T} \tilde{\mathbf{m}} \dot{\tilde{\mathbf{q}}}-\delta \widetilde{\mathbf{q}}^{T} \tilde{\mathbf{k}} \tilde{\mathbf{q}}-\delta \widetilde{\mathbf{q}}^{T} \hat{\mathbf{F}}_{w}\right) d t \\
& -\int_{t_{1}}^{t_{2}}\left(\delta \tilde{\mathbf{q}}^{T} \mathbf{K}_{h w}^{T} \ddot{\mathbf{q}}+\delta \ddot{\mathbf{q}}^{T} \mathbf{K}_{h w} \tilde{\mathbf{q}}+\delta \ddot{\mathbf{Q}}^{T} \mathbf{K}_{s w} \tilde{\mathbf{q}}+\delta \tilde{\mathbf{q}}^{T} \mathbf{K}_{s w}^{T} \ddot{\mathbf{Q}}\right) d t,
\end{aligned}
$$

from which, when integrating by parts and vanishing the two time terminal variations, it follows

$$
\begin{aligned}
& \delta H_{s f}[\tilde{\mathbf{q}}, \mathbf{q}, \mathbf{Q}]=\int_{t_{1}}^{t_{2}}\left\{\delta \ddot{\mathbf{Q}}^{T}[\mathbf{M} \ddot{\mathbf{Q}}+\mathbf{K Q}]+\left[\delta \ddot{\mathbf{Q}}^{T} \quad \delta \dot{\mathbf{q}}^{T}\left[\begin{array}{ll}
\mathbf{K}_{s s} & \mathbf{K}_{s h} \\
\mathbf{K}_{h s} & \mathbf{K}_{h h}
\end{array}\right]\left[\begin{array}{l}
\mathbf{Q} \\
\mathbf{q}
\end{array}\right]\right\} d t\right. \\
& +\int_{t_{1}}^{t_{2}} \delta \dot{\mathbf{q}}^{T}\left[\mathbf{m} \ddot{\mathbf{q}}+\left(\mathbf{k}+\mathbf{K}_{g}\right) \mathbf{q}-\hat{\mathbf{F}}_{h}\right] d t-\int_{t_{1}}^{t_{2}} \delta \widetilde{\mathbf{q}}^{T}\left(\tilde{\mathbf{m}} \ddot{\tilde{\mathbf{q}}}+\tilde{\mathbf{k}} \widetilde{\mathbf{q}}+\hat{\mathbf{F}}_{w}\right) d t \\
& -\int_{t_{1}}^{t_{2}}\left(\delta \widetilde{\mathbf{q}}^{T} \mathbf{K}_{h w}^{T} \ddot{\mathbf{q}}+\delta \ddot{\mathbf{q}}^{T} \mathbf{K}_{h w} \tilde{\mathbf{q}}+\delta \ddot{\mathbf{Q}}^{T} \mathbf{K}_{s w} \tilde{\mathbf{q}}+\delta \widetilde{\mathbf{q}}^{T} \mathbf{K}_{s w}^{T} \ddot{\mathbf{Q}}\right) d t .
\end{aligned}
$$

Since the variations $\delta \ddot{\mathbf{Q}}, \delta \ddot{\mathbf{q}}$ and $\delta \widetilde{\mathbf{q}}$ are independent, from $\delta H_{s f}=0$ it follows

$$
\left[\begin{array}{ccc}
\mathbf{M} & 0 & 0 \\
0 & \mathbf{m} & 0 \\
\mathbf{K}_{s w}^{T} & \mathbf{K}_{h w}^{T} & \tilde{\mathbf{m}}
\end{array}\right]\left[\begin{array}{c}
\ddot{\mathbf{Q}} \\
\ddot{\mathbf{q}} \\
\ddot{\tilde{\mathbf{q}}}
\end{array}\right]+\left[\begin{array}{ccc}
\mathbf{K}+\mathbf{K}_{s s} & \mathbf{K}_{s h} & -\mathbf{K}_{s w} \\
\mathbf{K}_{h s} & \mathbf{k}+\mathbf{k}_{g}+\mathbf{K}_{h h} & -\mathbf{K}_{h w} \\
0 & 0 & \tilde{\mathbf{k}}
\end{array}\right]\left[\begin{array}{c}
\mathbf{Q} \\
\mathbf{q} \\
\tilde{\mathbf{q}}
\end{array}\right]=\left[\begin{array}{c}
0 \\
\hat{\mathbf{F}}_{h} \\
-\hat{\mathbf{F}}_{w}
\end{array}\right]
$$

This is the numerical equation describing the dynamics of the integrated system. The degree of freedom of the system depends on the retaining mode number of each substructure / subdomain, and generally it equals $n+N+\tilde{n}$. In this equation, the matrices $\mathbf{M}, \mathbf{m}, \tilde{\mathbf{m}}, \mathbf{K}, \mathbf{k}$ and $\tilde{\mathbf{k}}$ are diagonal. In this equation, the force vector $\hat{\mathbf{F}}_{h}$ is generated from the external forces applied to the hull structure and the force vector $\hat{\mathbf{F}}_{w}$ is generated the external forces applied to the water, such as the incident wave acceleration. 

symmetrical, which can be symmetrised by using one of symmetrisation methods (Xing, 1991; Xing, 1996), we derive the following symmetrical equation

$$
\left[\begin{array}{cc}
\overline{\mathbf{K}} & \mathbf{0} \\
\mathbf{0} & \tilde{\mathbf{m}}
\end{array}\right]\left[\begin{array}{c}
\ddot{\mathbf{Q}} \\
\ddot{\tilde{\mathbf{q}}}
\end{array}\right]+\left[\begin{array}{cc}
\overline{\mathbf{K M}}^{-1} \overline{\mathbf{K}} & -\overline{\mathbf{K M}}^{-1} \mathbf{R} \\
-\mathbf{R}^{T} \overline{\mathbf{M}}^{-1} \overline{\mathbf{K}} & \tilde{\mathbf{k}}+\mathbf{R}^{T} \overline{\mathbf{M}}^{-1} \mathbf{R}
\end{array}\right]\left[\begin{array}{c}
\overline{\mathbf{Q}} \\
\tilde{\mathbf{q}}
\end{array}\right]=\left[\begin{array}{c}
\overline{\mathbf{K} \bar{M}^{-1} \overline{\mathbf{F}}} \\
\overline{\mathbf{f}}-\mathbf{R}^{T} \overline{\mathbf{M}}^{-1} \overline{\mathbf{F}}
\end{array}\right],
$$

where

$$
\begin{aligned}
& \overline{\mathbf{M}}=\left[\begin{array}{cc}
\mathbf{M} & 0 \\
0 & \mathbf{m}
\end{array}\right], \quad \overline{\mathbf{K}}=\left[\begin{array}{cc}
\mathbf{K}+\mathbf{K}_{s s} & \mathbf{K}_{s h} \\
\mathbf{K}_{h s} & \mathbf{k}+\mathbf{k}_{g}+\mathbf{K}_{h h}
\end{array}\right], \quad \overline{\mathbf{Q}}=\left[\begin{array}{l}
\mathbf{Q} \\
\mathbf{q}
\end{array}\right], \\
& \mathbf{R}=\left[\begin{array}{l}
\mathbf{K}_{s w} \\
\mathbf{K}_{h w}
\end{array}\right], \quad \overline{\mathbf{F}}=\left[\begin{array}{c}
0 \\
\hat{\mathbf{F}}_{h}
\end{array}\right], \quad \overline{\mathbf{f}}=-\hat{\mathbf{F}}_{w} .
\end{aligned}
$$

\section{Dynamic interaction analysis of the coupling system}

Based on the equations developed in section 5, we can now carry on a numerical analysis of the dynamic interaction of the integrated system. We are more interested in the effect of the hull motion on the propulsion system, so that we define some parameters to measure it.

6.1 Natural vibrations and shaft frequency / deformation factors

Natural vibrations. The natural frequencies and the corresponding modes of the coupling system are governed by equation

$$
\left[\begin{array}{cc}
\overline{\mathbf{K}} & \mathbf{0} \\
\mathbf{0} & \tilde{\mathbf{m}}
\end{array}\right]\left[\begin{array}{c}
\ddot{\mathbf{Q}} \\
\ddot{\tilde{\mathbf{q}}}
\end{array}\right]+\left[\begin{array}{cc}
\overline{\mathbf{K}}^{-1} \overline{\mathbf{K}} & -\overline{\mathbf{K}}^{-1} \mathbf{R} \\
-\mathbf{R}^{T} \overline{\mathbf{M}}^{-1} \overline{\mathbf{K}} & \tilde{\mathbf{k}}+\mathbf{R}^{T} \overline{\mathbf{M}}^{-1} \mathbf{R}
\end{array}\right]\left[\begin{array}{c}
\overline{\mathbf{Q}} \\
\tilde{\mathbf{q}}
\end{array}\right]=0,
$$

derived by vanishing the left side force vector in Eq. (51). Mathematically, this is an eigenvalue problem, which can be solved using some well-designed software. There might exist zero frequency of this problem, therefore the frequency shift technique should be used to obtain the solutions (Xing, 1991; Xing, 1996).

Assume that the first $\widehat{N}$ natural frequencies $\widehat{\Omega}_{I}$ and corresponding natural modes $\widehat{\Phi}_{I}$ are obtained, which are represented in a matrix form

$$
\widehat{\boldsymbol{\Lambda}}=\operatorname{diag}\left(\widehat{\Omega}_{1}, \widehat{\Omega}_{2}, \cdots, \widehat{\Omega}_{\hat{N}}\right), \quad \widehat{\boldsymbol{\Phi}}=\left[\begin{array}{llll}
\widehat{\Phi}_{1} & \widehat{\Phi}_{2} & \cdots & \widehat{\Phi}_{\hat{N}}
\end{array}\right],
$$

which satisfy the orthogonal relationships 


$$
\widehat{\boldsymbol{\Phi}}^{T}\left[\begin{array}{cc}
\overline{\mathbf{K}} & \mathbf{0} \\
\mathbf{0} & \tilde{\mathbf{m}}
\end{array}\right] \widehat{\boldsymbol{\Phi}}=\mathbf{I}, \quad \widehat{\boldsymbol{\Phi}}^{T}\left[\begin{array}{cc}
\overline{\mathbf{K}}^{-1} \overline{\mathbf{K}} & -\overline{\mathbf{K M}}^{-1} \mathbf{R} \\
-\mathbf{R}^{T} \overline{\mathbf{M}}^{-1} \overline{\mathbf{K}} & \tilde{\mathbf{k}}+\mathbf{R}^{T} \overline{\mathbf{M}}^{-1} \mathbf{R}
\end{array}\right] \widehat{\boldsymbol{\Phi}}=\operatorname{diag}\left(\widehat{\Omega}_{I}\right) .
$$

372

Shaft frequency factors. To obtain the shaft motion as smaller as possible, we need to avoid the natural frequency of the shaft substructure far from the natural frequencies of the integrated system. To measure this, we define the shaft frequency factors

$$
\xi_{J}^{(I)}=\Omega_{J} / \widehat{\Omega}_{I}, \quad(I=1,2, \cdots, \widehat{N} ; \quad J=1,2, \cdots, N),
$$

representing the ratio of the frequency $\Omega_{J}$ of the shaft substructure over the frequency $\widehat{\Omega}_{I}$ of the integrated system. According to frequency rule for substructure methods (Li, 2013), we requires

$$
\xi_{J}^{(I)}=\left\{\begin{array}{ll}
\leq 1 / 3 \sim 1 / 2, & \Omega_{J}<\widehat{\Omega}_{I} \\
\geq 2 \sim 3, & \Omega_{J}>\widehat{\Omega}_{I}
\end{array}, \quad(I=1,2, \cdots, \widehat{N} ; \quad J=1,2, \cdots, N),\right.
$$

to avoid resonance between the substructure and the integrated system. This implies that the shaft frequency factors should be far from 1 to avoid any resonances.

Shaft deformation factors. The mode shape $\widehat{\Phi}_{I}$ of the integrated coupling system defines the following vector

$$
\widehat{\Phi}_{I}=\left[\begin{array}{cc}
\widehat{\mathbf{Q}}^{T} & \widehat{\widetilde{\mathbf{q}}}^{T}
\end{array}\right]_{I}^{T}=\left[\begin{array}{lll}
\widehat{\mathbf{Q}}^{T} & \widehat{\mathbf{q}}^{T} & \widehat{\widetilde{\mathbf{q}}}^{T}
\end{array}\right]_{I}^{T},
$$

from which the corresponding components of each substructure / subdomain can be obtained using Eqs. (30), (37) and (44), i.e.

$$
\begin{array}{ll}
\widehat{\mathbf{u}}^{(I)}=\boldsymbol{\varphi} \widehat{\mathbf{q}}^{(I)}, & \widehat{\mathbf{q}}^{(I)}=\left[\begin{array}{llll}
\widehat{q}_{1}^{(I)} & \widehat{q}_{2}^{(I)} & \cdots & \widehat{q}_{n}^{(I)}
\end{array}\right]^{T}, \\
\widehat{\mathbf{U}}^{(I)}=\boldsymbol{\Phi} \widehat{\mathbf{Q}}^{(I)}, & \widehat{\mathbf{Q}}^{(I)}=\left[\begin{array}{lllll}
\widehat{Q}_{1}^{(I)} & \widehat{Q}_{2}^{(I)} & \cdots & \widehat{Q}_{N}^{(I)}
\end{array}\right]^{T}, \\
\hat{p}^{(I)}=\boldsymbol{\Psi} \overline{\mathbf{q}}^{(I)}, & \overline{\mathbf{q}}^{(I)}=\left[\begin{array}{lllll}
\widetilde{\widetilde{q}}_{1}^{(I)} & \overline{\tilde{q}}_{2}^{(I)} & \cdots & \overline{\tilde{q}}_{\tilde{n}}^{(I)}
\end{array}\right]^{T} .
\end{array}
$$

Physically, these components represent the hull displacement, the shaft displacement and the water pressure for the I-th mode of the integrated system.

Involving the propulsion shaft unit, we wish the deformation of the shaft relative to the hull motion would be small, so that the deformation of the shaft unit in the I-th mode of the integrated system is small and the bearings can safety operation. The J-th bearing connected to the shaft at point $A_{J}\left(\mathbf{Y}_{J}^{A}\right)$ 
392

393

394

395

396

and to the hull at point $B_{J}\left(\mathbf{Y}_{J}^{B}\right)$, so that the displacement of point $A_{J}\left(\mathbf{Y}_{J}^{A}\right)$ relative to point $B_{J}\left(\mathbf{Y}_{J}^{B}\right)$ in the I-th mode of the integrated system is $\mathbf{U}_{I}^{A}\left(\mathbf{Y}_{J}^{A}\right)-\mathbf{u}_{I}^{B}\left(\mathbf{Y}_{J}^{B}\right)$. Therefore we define a shaft relative motion factor for the I-th mode of the integrated system as the ratio of the averaged relative displacement of the shaft points $A_{J}\left(\mathbf{Y}_{J}^{A}\right)$ of all bearings over the corresponding averaged hull displacement at points $B_{J}\left(\mathbf{Y}_{J}^{B}\right)$, that is

$$
\gamma_{I}=\frac{\sum_{J}^{\hat{I}}\left|\mathbf{U}_{I}^{A}\left(\mathbf{Y}_{J}^{A}\right)-\mathbf{u}_{I}^{B}\left(\mathbf{Y}_{J}^{B}\right)\right|}{\sum_{J}^{\hat{I}}\left|\mathbf{u}_{I}^{B}\left(\mathbf{Y}_{J}^{B}\right)\right|}, \quad I=1,2, \cdots, \hat{N}
$$

Considering all modes, we define the shaft relative motion factor vector

$$
\boldsymbol{\gamma}=\left[\begin{array}{llll}
\gamma_{1} & \gamma_{2} & \cdots & \gamma_{\hat{N}}
\end{array}\right\rfloor
$$

To design a more suitable arrangement of the propulsion shat unit, we need to choose a smaller value of the shaft relative motion factor vector, i.e.

$$
|\gamma|=\sqrt{\gamma^{T} \gamma}
$$

The smallest shaft relative motion factor vector cannot be zero, because the elastic shaft is supported by elastic bearings, so that the relative motion of the elastic shaft does not vanish. Eq. (56) and Eq. (62) provide two parameters to measure the dynamic coupling level of the shat unit and the hull structure from the natural vibrations.

\subsection{Dynamic responses}

Mode summation solution. The dynamic responses of the integrated can be calculated by solving Eq. (51). The mode summation method provides a fast way to obtain the solution of Eq. (51). We denote the dynamic response of the integrated system in a mode summation form

$$
\left[\begin{array}{c}
\overline{\mathbf{Q}} \\
\tilde{\mathbf{q}}
\end{array}\right]=\widehat{\boldsymbol{\Phi}} \breve{\mathbf{Q}},
$$

from which, when substituted into Eq. (51) using the orthogonal relationships (55), it follows 


$$
\ddot{\widetilde{\mathbf{Q}}}+\operatorname{diag}\left(\widehat{\Omega}_{I}^{2}\right) \breve{\mathbf{Q}}=\breve{\mathbf{F}}, \quad \breve{\mathbf{F}}=\widehat{\mathbf{\Phi}}\left[\begin{array}{c}
\overline{\mathbf{K}} \overline{\mathbf{M}}^{-1} \overline{\mathbf{F}} \\
\overline{\mathbf{f}}-\mathbf{R}^{T} \overline{\mathbf{M}}^{-1} \overline{\mathbf{F}}
\end{array}\right],
$$

414 which consists of $n+N+\tilde{n}$ independent dynamic equations describing the dynamic responses of 415 the integrated system excited by the external forces. To consider practical damping effect, we introduce the damping matrix as follows (Bathe, 1996)

$$
\begin{aligned}
& \widehat{\mathbf{C}}=\alpha\left[\begin{array}{cc}
\overline{\mathbf{K}} & \mathbf{0} \\
\mathbf{0} & \tilde{\mathbf{m}}
\end{array}\right]+\beta\left[\begin{array}{cc}
\overline{\mathbf{K M}}^{-1} \overline{\mathbf{K}} & -\overline{\mathbf{K}}^{-1} \mathbf{R} \\
-\mathbf{R}^{T} \overline{\mathbf{M}}^{-1} \overline{\mathbf{K}} & \tilde{\mathbf{K}}+\mathbf{R}^{T} \overline{\mathbf{M}}^{-1} \mathbf{R}
\end{array}\right], \\
& \breve{\mathbf{C}}=\widehat{\boldsymbol{\Phi}}^{T} \widehat{\mathbf{C}} \widehat{\mathbf{\Phi}}=\alpha \mathbf{I}+\beta \operatorname{diag}\left(\widehat{\Omega}_{I}^{2}\right)=\operatorname{diag}\left(\breve{C}_{I}\right),
\end{aligned}
$$

where the coefficients $\alpha$ and $\beta$ can be determined by using available practical experiment / experience data. The dynamic equation including damping effect is now written as

$$
\ddot{\breve{\mathbf{Q}}}+\operatorname{diag}\left(\breve{C}_{I}\right) \dot{\overline{\mathbf{Q}}}+\operatorname{diag}\left(\widehat{\Omega}_{I}^{2}\right) \breve{\mathbf{Q}}=\breve{\mathbf{F}} .
$$

The equation for the I-th independent mode is written as

$$
\ddot{\breve{Q}}_{I}+\breve{C}_{I} \dot{\grave{Q}}_{I}+\widehat{\Omega}_{I}^{2} \breve{Q}_{I}=\breve{F}_{I} .
$$

Generally, this equation can be solved using a time integration method (Bathe, 1983; Zienkiewicz, 1991). If the external force is a sinusoidal force of frequency $\breve{\omega}, \breve{F}_{I}=\breve{f}_{I} e^{-\mathrm{j} \breve{\omega} t}$, the solution of Eq. (67) has a form $\breve{Q}_{I}=\breve{q}_{I} e^{-\mathrm{j} \breve{\omega t}}$, so that

$$
\begin{aligned}
& \left(1-\breve{\eta}_{I}^{2}-2 \mathrm{j} \breve{\eta}_{I}\right) \breve{q}_{I}=\breve{f}_{I} / \widehat{\Omega}_{I}^{2}, \quad \breve{\eta}_{I}=\breve{\omega} / \widehat{\Omega}_{I}, \quad \varsigma_{I}=\breve{C}_{I} /\left(2 \widehat{\Omega}_{I}\right), \\
& \breve{q}_{I}=\frac{\breve{f}_{I} / \widehat{\Omega}_{I}^{2}}{\left(1-\breve{\eta}_{I}^{2}-2 \mathrm{j} \breve{\eta}_{I}\right)}=\left|\breve{q}_{I}\right| e^{-\mathrm{j}\left(\breve{\omega}_{\left.\mathrm{t}-\breve{\phi}_{I}\right)}\right.}, \quad\left|\breve{q}_{I}\right|=\frac{\breve{f}_{I} / \widehat{\Omega}_{I}^{2}}{\sqrt{\left.\left(1-\breve{\eta}_{I}^{2}\right)^{2}+4 \breve{\eta}_{I}^{2}\right)}}, \quad \breve{\phi}_{I}=\tan ^{-1} \frac{2 \breve{\eta}_{I}}{1-\breve{\eta}_{I}^{2}} .
\end{aligned}
$$

Physical dynamic responses. Obtaining the generalised coordinate vector $\breve{\mathbf{Q}}$, we can calculate the corresponding generalised vectors $\mathbf{Q}, \mathbf{q}$ and $\widetilde{\mathbf{q}}$ for substructures from Eq. (63), so that the dynamic displacement / pressure responses at each point of substructures / subdomain: shaft, hull and water can be calculated from Eq. (37), (30) and (44), respectively. To investigate the effect of hull motion with water interaction on the propulsion unit, we can calculated the shaft relative motion factor to the excitation force frequency $\breve{\omega}$ in a similar form as Eq. (60), i.e. 


$$
\gamma_{\omega}=\frac{\sum_{J}^{\hat{I}}\left|\mathbf{U}_{\omega}^{A}\left(\mathbf{Y}_{J}^{A}\right)-\mathbf{u}_{\omega}^{B}\left(\mathbf{Y}_{J}^{B}\right)\right|}{\sum_{J}^{\hat{I}}\left|\mathbf{u}_{\omega}^{B}\left(\mathbf{Y}_{J}^{B}\right)\right|} .
$$

Also, we define a shaft twist factor

$$
\alpha_{\omega}=\max \left|\mathbf{U}\left(Y_{J}^{A}\right)-\mathbf{U}(0)\right|
$$

Physically, Eq. (69) gives the ration of the averaged shaft displacement over the averaged hull displacement, and Eq. (70) represents the displacement at the shaft point $Y_{J}^{A}$ of bearing $\mathrm{J}$ relative to the origin $\hat{O}$ of the shaft coordinate system which reflects the rotation of the shaft. Therefore, these two factors measure the motion and deformation of the shaft. For example, if Eq. (69) equals zero, it implies that the shaft undergoes a rigid translation with the hull base. Large values of these factors should be avoided for shaft safe operations.

\section{An example}

In this section, based on the generalised theory and analysis approach, we investigate a 2dimensional (2-D) simplified example. As shown in Fig. 5, we consider the ship hull as a 2-D beam of length $L_{h}$ beam, mass density $\rho_{h}$, section area $S_{h}$ and bending stiffness $E_{h} J_{h}$ floating on the water of depth $\mathrm{H}$ and width $L_{w}$. On the boundary $\Gamma_{w}$ of the water, there is an incident acceleration wave $\hat{w}_{1}=\left(1+x_{3} / H\right) \cos \breve{\omega} t$ in the $x_{1}$ direction. This assumption case may be considered as the one in which a ship is moored in a port and subjected a sea wave excitation. The propulsion unit is another uniform 2-D beam (neglecting masses of propeller / disk) of length $L$, mass density $\rho$, section area $S$ and bending stiffness $E J$ fixed at two points $B_{1}\left(X_{11}^{B}\right)$ and $B_{2}\left(X_{21}^{B}\right)$ on the ship hull by two bearings $A_{1}\left(Y_{11}^{A}\right)$ and $A_{2}\left(Y_{21}^{A}\right)$ of vertical stiffness $k_{13}$ and $k_{23}$, respectively. We aim to investigate the interactions of vertical bending motions of two beams, therefore only their bending deformation in the vertical direction is considered. For our convenience, we assume that the central line of the floating beam is on the static free surface plane and the coordinate system $o-x_{1} x_{2} x_{3}$ is fixed at the middle point $\mathrm{o}$ on the static equilibrium free surface, although it is located at a general point. We 


$$
\begin{aligned}
& X_{I i}^{A}=X_{i 0}+Y_{I i}^{A}, \quad X_{I i}^{B}=X_{i 0}+Y_{I i}^{B}, \quad I=1,2, \\
& \mathbf{K}_{A}^{\Delta}=k_{13} \Delta\left(Y_{1}-Y_{11}^{A}\right)+k_{23} \Delta\left(Y_{1}-Y_{21}^{A}\right), \\
& \mathbf{K}_{B}^{\Delta}=k_{13} \Delta\left(X_{1}-X_{11}^{B}\right)+k_{23} \Delta\left(X_{1}-X_{21}^{B}\right), \\
& \mathbf{F}_{s f}^{\Delta}=0, \quad \overline{\mathbf{K}}_{B}^{\Delta}=\mathbf{K}_{B}^{\Delta}=\overline{\mathbf{K}}_{B E}^{\Delta}, \quad \overline{\mathbf{K}}_{A}^{\Delta}=\mathbf{K}_{A}^{\Delta}=\overline{\mathbf{K}}_{A E}^{\Delta} .
\end{aligned}
$$

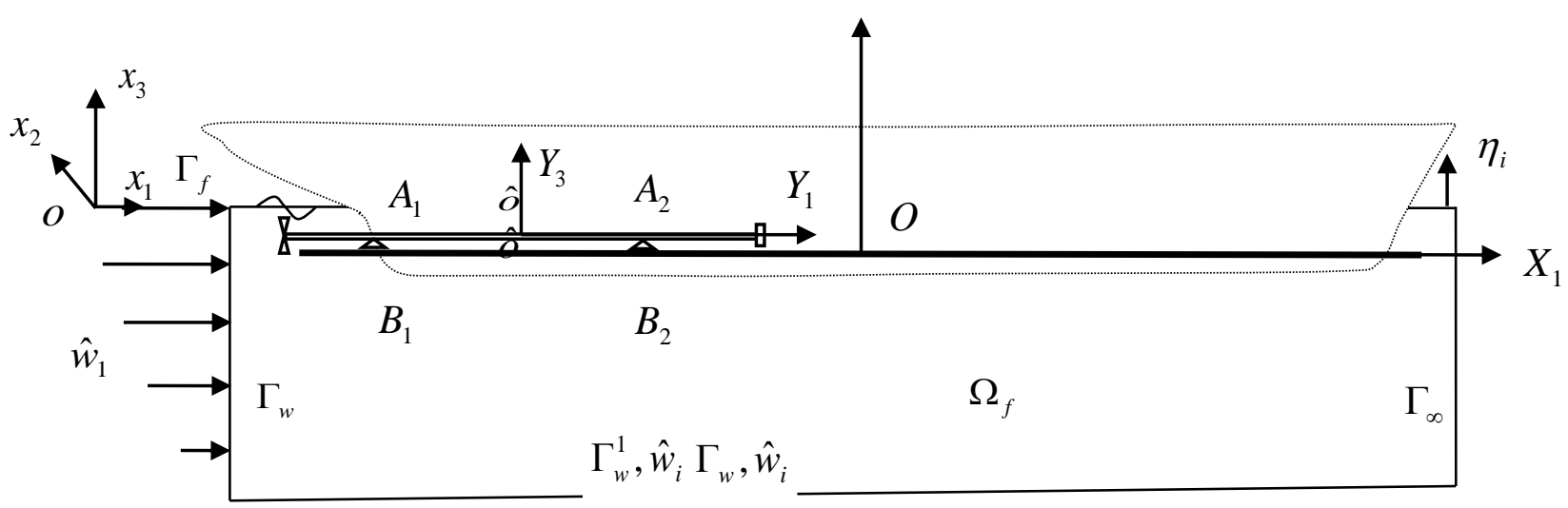

461

Fig. 5. A 2-D water-shaft beam-hull beam interaction system with boundary conditions: $p=0$ on

$\Gamma_{\infty} ; \hat{w}_{i}=0$ on $\Gamma_{w}^{1}$ of length $L_{w}$ and $\hat{w}_{1}=\left(1+x_{3} / H\right) \cos \breve{\omega} t$ on $\Gamma_{w}$ of height $H$.

\subsection{Mode functions of two beams}

To analyse this simplified model, we can use theoretical mode functions of free-free beam given as

follows

$$
f_{J}(\varsigma)=\left\{\begin{array}{cc}
1 / 2 & J=1 \\
\frac{1}{2}\left\{\frac{\cosh \left(\varsigma \mu_{J}\right)}{\cosh \mu_{J}}+\frac{\cos \left(\varsigma \mu_{J}\right)}{\cos \mu_{J}}\right\} & J=3,5, \ldots
\end{array},\right.
$$

$$
f_{J}(\varsigma)=\left\{\begin{array}{cc}
\sqrt{3} \varsigma / 2 & J=2 \\
\frac{1}{2}\left\{\frac{\sinh \left(\varsigma \mu_{J}\right)}{\sinh \mu_{J}}+\frac{\sin \left(\varsigma \mu_{J}\right)}{\sin \mu_{J}}\right\} & J=4,6, \ldots
\end{array},\right.
$$


where $\varsigma=X / a$ and $a$ is the half length of beam and $\mu_{J}$ denote positive real roots of the eigenvalue equation

$$
\begin{cases}\tan \mu_{J}+\tanh \mu_{J}=0 & J=1,3,5, \ldots \\ \tan \mu_{J}-\tanh \mu_{J}=0 & J=2,4,6, \ldots\end{cases}
$$

The orthogonal condition of these mode functions is given by

$$
\int_{-1}^{1} f_{I}(\varsigma) f_{J}(\varsigma) d \varsigma=\left\{\begin{array}{cc}
0 & I \neq J \\
1 / 2 & I=J
\end{array}\right. \text {. }
$$

In these mode functions, physically, $f_{1}(\varsigma)$ and $f_{2}(\varsigma)$ are two rigid modes of zero frequency and function $f_{3}(\varsigma)$ is the first symmetrical bending mode of frequency $\omega$ for hull and $\Omega$ for shaft. In the numerical works, the first five modes will be chosen to represent two beam motions. Following Eqs. (30), (37) and (47), now we can represent the vertical motions of hull / shaft beams respectively in the forms

$$
\begin{aligned}
& u_{3}=\boldsymbol{\varphi q}, \quad \boldsymbol{\varphi}=\left[\begin{array}{lll}
f_{1} & \cdots & f_{5}
\end{array}\right], \quad \mathbf{q}=\left[\begin{array}{lll}
q_{1} & \cdots & q_{5}
\end{array}\right]^{T,} \quad \boldsymbol{\varsigma}=X_{1} / a, \quad a=L_{h} / 2, \\
& m=\rho_{h} S_{h} L_{h} / 4, \quad \mathbf{m}=m \mathbf{I}, \quad \mathbf{k}=\mathbf{m} \lambda^{2}, \quad \lambda^{2}=\operatorname{diag}\left(0,0, \omega^{2}\right),
\end{aligned}
$$

$U_{3}=\boldsymbol{\Phi} \mathbf{Q}, \quad \boldsymbol{\Phi}=\left[\begin{array}{lll}f_{1} & \cdots & \cdots_{3}\end{array}\right], \quad \mathbf{Q}=\left[\begin{array}{lll}Q_{1} & \cdots & Q_{3}\end{array}\right]^{T}, \quad \varsigma=Y_{1} / a, \quad a=L / 2$, $M=\rho S L / 4, \quad \mathbf{M}=M \mathbf{I}, \quad \mathbf{K}=\mathbf{M} \mathbf{\Lambda}^{2}, \quad \boldsymbol{\Lambda}^{2}=\operatorname{diag}\left(0,0, \Omega^{2}\right)$,

$$
\begin{aligned}
& {\left[\begin{array}{ll}
\mathbf{K}_{s s} & \mathbf{K}_{s h} \\
\mathbf{K}_{h s} & \mathbf{K}_{h h}
\end{array}\right]=\int_{-1}^{1}\left[\begin{array}{cc}
\boldsymbol{\Phi}^{T} & \mathbf{0} \\
\mathbf{0} & \boldsymbol{\varphi}^{T}
\end{array}\right]\left[\begin{array}{cc}
\mathbf{K}_{A}^{\Delta} & -\mathbf{K}_{A}^{\Delta} \\
-\mathbf{K}_{B}^{\Delta} & \mathbf{K}_{B}^{\Delta}
\end{array}\right]\left[\begin{array}{cc}
\mathbf{\Phi} & \mathbf{0} \\
\mathbf{0} & \boldsymbol{\varphi}
\end{array}\right] d \varsigma} \\
& \mathbf{K}_{s s}=\boldsymbol{\Phi}^{T}\left(Y_{11}^{A}\right) k_{13} \boldsymbol{\Phi}\left(Y_{11}^{A}\right)+\boldsymbol{\Phi}^{T}\left(Y_{21}^{A}\right) k_{23} \boldsymbol{\Phi}\left(Y_{21}^{A}\right), \\
& \mathbf{K}_{s h}=-\boldsymbol{\Phi}^{T}\left(Y_{11}^{A}\right) k_{13} \boldsymbol{\varphi}\left(X_{11}^{B}\right)-\boldsymbol{\Phi}^{T}\left(Y_{21}^{A}\right) k_{23} \boldsymbol{\varphi}\left(X_{21}^{B}\right)=\mathbf{K}_{h s}, \\
& \mathbf{K}_{h h}=\boldsymbol{\varphi}^{T}\left(X_{11}^{B}\right) k_{13} \boldsymbol{\varphi}\left(X_{11}^{B}\right)+\boldsymbol{\varphi}^{T}\left(X_{21}^{B}\right) k_{23} \boldsymbol{\varphi}\left(X_{21}^{B}\right) .
\end{aligned}
$$

Here, in Eq. (75-3), the integrations with respect to non-dimensional length $\varsigma$ involve Delta functions to be non-dimensional, and therefore the resultant matrices have dimension of stiffness.

\subsection{Mode functions of 2-D water domain}

For the water domain, we consider it as 2-D incompressible fluid $(c \rightarrow \infty)$ of depth $\mathrm{H}$ and width $L_{w}$. By using a separation method of variables, we obtain the mode functions of this 2-D water domain as follows. 


$$
\tilde{\omega}_{I}^{2}=\tilde{\omega}_{n+1}^{2}=\frac{n \pi g}{L_{w}} \tanh \frac{n \pi H}{L_{w}}, \quad I=n+1,
$$

$$
\Psi_{I}=\Psi_{n+1}=\left\{\begin{array}{cl}
1 / \sqrt{2}, & n=0, \\
\cos \frac{n \pi x_{1}}{L_{w}} \cosh \frac{n \pi\left(x_{3}+H\right)}{L_{w}}, & n=2,4, \cdots . \\
\sin \frac{n \pi x_{1}}{L_{w}} \cosh \frac{n \pi\left(x_{3}+H\right)}{L_{w}}, & n=1,3,5, \cdots
\end{array}\right.
$$

$$
\left.\frac{1}{\rho_{f}} \int_{\Omega_{f}} \Psi_{I, i}^{T} \Psi_{J, i}\right) d \Omega_{f}=\left\{\begin{array}{cc}
0, & I \neq J \\
\tilde{k}_{I}, & I=J
\end{array}, \quad \tilde{\boldsymbol{k}}=\operatorname{diag}\left(\tilde{k}_{I}\right), \quad\left[\mathrm{m}^{5} \mathrm{~N}^{-1} \mathrm{~S}^{-2}\right],\right.
$$

$$
\frac{1}{\rho_{f} g} \int_{\Gamma_{f}} \Psi_{I}^{T} \Psi_{I} d \Gamma=\left\{\begin{array}{cc}
0, & I \neq J \\
\tilde{m}_{I}, & I=J
\end{array}, \quad \tilde{\boldsymbol{m}}=\operatorname{diag}\left(\tilde{m}_{I}\right), \quad\left[\mathrm{m}^{5} \mathrm{~N}^{-1}\right],\right.
$$
$\tilde{m}_{I}=\frac{L_{w} b}{2 \rho_{f} g} \cosh ^{2} \frac{n \pi H}{L_{w}}, \quad b=1 \quad$ unit thickness in $x_{2}$ direction .

490

491

492

493

494

495

496

497

498

499

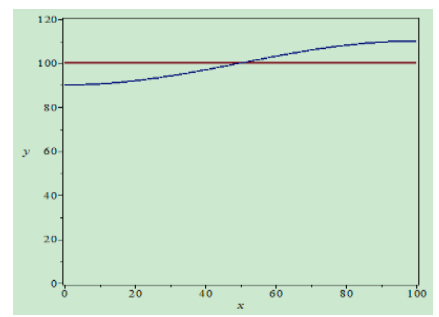

$\operatorname{Mode} 2(n=1)$

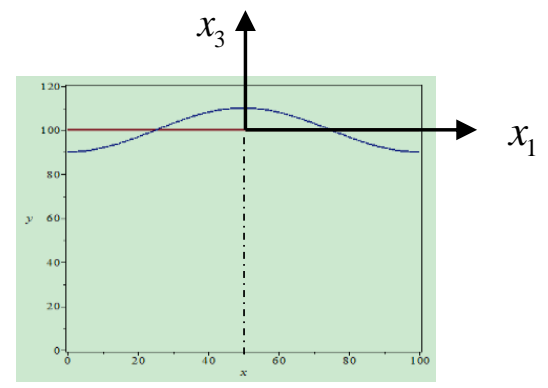

Mode $3(\mathrm{n}=2)$

Mode $1(\mathrm{n}=0)$

Fig. 6. The first three modes of the 2-D incompressible water domain of depth $H=100$ in direction $y=x_{3}$ and width $L_{w}=100$ in direction $x=x_{1}$. The position of coordinate $o-x_{1} x_{2} x_{3}$ is given in mode 3, which are neglected for other two modes.

The first three modes for the water domain $H=100$ and $L_{w}=100$ are shown in Fig. 6 in which the first one is a constant pressure mode with zero frequency, the second one plays a slash form while the third one characterizes an cosine wave pattern. To model the water pressure, we will use it first 5 modes for mode summation, so that from (42-47) it follows

$$
\tilde{\lambda}=\operatorname{diag}\left(\tilde{\omega}_{1}, \cdots, \tilde{\omega}_{5}\right), \quad \boldsymbol{\Psi}=\left[\begin{array}{lll}
\Psi_{1} & \ldots & \Psi_{5}
\end{array}\right], \quad \tilde{\mathbf{q}}=\left[\begin{array}{lll}
\tilde{q}_{1} & \cdots & \tilde{q}_{5}
\end{array}\right]^{T}, \quad \tilde{n}=5 .
$$




$$
\mathbf{K}_{s w}=\mathbf{0}, \quad \mathbf{K}_{h w}=\int_{-L_{h} / 2}^{L_{h} / 2} \boldsymbol{\varphi}^{T} \boldsymbol{\Psi} d X_{1}, \quad\left[\mathrm{~m}^{2}\right],
$$

$$
\hat{\mathbf{F}}_{h}=\mathbf{0}, \quad \hat{\mathbf{F}}_{w}=\int_{0}^{H} \boldsymbol{\Psi}^{T} \hat{w}_{1} d x_{3}=\cos \breve{\omega} t \int_{0}^{H} \boldsymbol{\Psi}^{T}\left(1+x_{3} / H\right) d x_{3}, \quad\left[\mathrm{~m}^{3} \mathrm{~S}^{-2}\right] .
$$

501

502

503

504

505

506

507

508

509

510

511

$$
\overline{\mathbf{M}}=\left[\begin{array}{cc}
\bar{M} \mathbf{I} & \mathbf{0} \\
\mathbf{0} & \mathbf{I}
\end{array}\right], \quad \overline{\mathbf{K}}=\left[\begin{array}{cc}
\overline{M \Lambda}^{2}+\overline{\mathbf{K}}_{s s} & \overline{\mathbf{K}}_{s h} \\
\overline{\mathbf{K}}_{h s} & \bar{\lambda}^{2}+\overline{\mathbf{k}}_{g}+\overline{\mathbf{K}}_{h h}
\end{array}\right],
$$

\subsection{Non-dimensional equations} length and mass, respectively, as well as define the following parameters,

where

To derive a non-dimensional equation, we choose $\omega^{-1}, L_{h}$ and $\mathrm{m}$ as the units to measure time,

$$
\begin{aligned}
& \overline{\mathbf{K}}_{s h}=\omega^{-2} m^{-1} \mathbf{K}_{s h}, \quad \overline{\mathbf{K}}_{s s}=\omega^{-2} m^{-1} \mathbf{K}_{s s}, \quad \overline{\mathbf{K}}_{h s}=\omega^{-2} m^{-1} \mathbf{K}_{h s}, \quad \overline{\mathbf{K}}_{h h}=\omega^{-2} m^{-1} \mathbf{K}_{h h}, \\
& \overline{\mathbf{K}}_{h w}=L_{h}^{-2} \mathbf{K}_{h w}, \quad \overline{\mathbf{Q}}=\mathbf{Q} / L_{h}, \quad \overline{\mathbf{q}}=\mathbf{q} / L_{h}, \quad \overline{\widetilde{\mathbf{q}}}=\tilde{\mathbf{q}} / \bar{p}, \quad \overline{\hat{\mathbf{F}}}_{w}=\hat{m}^{-1} \omega^{-2} \bar{p}^{-1} \hat{\mathbf{F}}_{w}, \\
& \overline{\boldsymbol{\lambda}}^{2}=\omega^{-2} \lambda^{2}, \quad \bar{\Lambda}^{2}=\omega^{-2} \Lambda^{2}, \quad \overline{\tilde{\lambda}}^{2}=\omega^{-2} \tilde{\lambda}^{2}, \quad \bar{p}=m \omega^{2} / L_{h}, \quad \hat{m}=\bar{p} / L_{h}^{3}, \\
& \overline{\tilde{\mathbf{m}}}=\tilde{\mathbf{m}} / \hat{m}, \quad \bar{M}=M / m, \quad \overline{\mathbf{k}}_{g}=\omega^{-2} m \mathbf{k}_{g} .
\end{aligned}
$$

from which Eq. (78) can be represented in the following non-dimensional form

$$
\left[\begin{array}{ccc}
\bar{M} \mathbf{I} & 0 & 0 \\
0 & \mathbf{I} & 0 \\
0 & \overline{\mathbf{K}}_{h w}^{T} & \overline{\overline{\mathbf{m}}}
\end{array}\right]\left[\begin{array}{c}
\ddot{\overline{\mathbf{Q}}} \\
\ddot{\overline{\mathbf{q}}} \\
\ddot{\widetilde{\mathbf{q}}}
\end{array}\right]+\left[\begin{array}{ccc}
\overline{M \Lambda}^{2}+\overline{\mathbf{K}}_{s s} & \overline{\mathbf{K}}_{s h} & 0 \\
\overline{\mathbf{K}}_{h s} & \bar{\lambda}^{2}+\overline{\mathbf{k}}_{g}+\overline{\mathbf{K}}_{h h} & -\overline{\mathbf{K}}_{h w} \\
0 & 0 & \overline{\tilde{\mathbf{m}}} \overline{\tilde{\lambda}}
\end{array}\right]\left[\begin{array}{c}
\overline{\mathbf{Q}} \\
\overline{\mathbf{q}} \\
\overline{\widetilde{\mathbf{q}}}
\end{array}\right]=\left[\begin{array}{c}
0 \\
0 \\
\overline{\hat{\mathbf{F}}_{w}}
\end{array}\right] .
$$

$$
\left[\begin{array}{cc}
\overline{\mathbf{K}} & \mathbf{0} \\
\mathbf{0} & \tilde{\mathbf{m}}
\end{array}\right]\left[\begin{array}{l}
\ddot{\tilde{\mathbf{Q}}} \\
\ddot{\tilde{\tilde{\mathbf{q}}}}
\end{array}\right]+\left[\begin{array}{cc}
\overline{\mathbf{K}}^{-1} \overline{\mathbf{K}} & -\overline{\mathbf{K}}^{-1} \mathbf{R} \\
-\mathbf{R}^{T} \overline{\mathbf{M}}^{-1} \overline{\mathbf{K}} & \overline{\tilde{\mathbf{m}}} \overline{\tilde{\lambda}}+\mathbf{R}^{T} \overline{\mathbf{M}}^{-1} \mathbf{R}
\end{array}\right]\left[\begin{array}{c}
\tilde{\mathbf{Q}} \\
\overline{\tilde{\mathbf{q}}}
\end{array}\right]=\left[\begin{array}{c}
\mathbf{0} \\
-\overline{\hat{\mathbf{F}}}_{w}
\end{array}\right],
$$

$$
\text { Eq. (79-3) is a matrix equation with } 15 \text { degrees of freedom, of which the numerical solution gives the }
$$
natural vibrations and the dynamic response of the system excited by the incident wave. For the numerical simulations, according to a practical ship configuration, we choose the following physical parameters and calculate the first 5 natural frequencies of the hull, shaft and the water domain, as 
follows.

518

519

520

522

523

525

526

527

528

530

531

532

533

534

535

536

537

538

539

540

541

Hull: $L_{h}=320 \mathrm{~m}, \rho_{h}=7820 S_{h}=1.086 \times 10^{6} \mathrm{kgm}^{-1}, E_{h}=2.06 \times 10^{11} \mathrm{Nm}^{-2}, J=1.535 \times 10^{3} \mathrm{~m}^{4}$,

$$
\lambda^{2}=\operatorname{diag}\left(\begin{array}{lllll}
0 & 0 & 13.90 & 105.60 & 406.100
\end{array}\right)
$$

Shaft: $L=14.4 \mathrm{~m}, r=0.4 \mathrm{~m}, S=\pi r^{2}, \rho=7820 S=3930 \mathrm{kgm}^{-1}, E=2.06 \times 10^{11} \mathrm{GNm}^{-2}$,

$$
k_{13}=k_{23}=1.0 \times 10^{9} \mathrm{Nm}^{-1}, \quad I=0.0201 \mathrm{~m}^{4}, \quad Y_{11}=-5 \mathrm{~m}, \quad Y_{21}=5 \mathrm{~m}, \quad X_{11}=-159 \mathrm{~m},
$$

$$
X_{21}=-149 \mathrm{~m}, \quad \Lambda^{2}=\operatorname{diag}\left(\begin{array}{lllll}
0 & 0 & 2.71 & 20.61 & 79.21
\end{array}\right) \times 10^{3} .
$$

Water: $\rho_{f}=1000 \mathrm{kgm}^{-3}, L_{w}=7 L_{h}, \quad H=200 \mathrm{~m}, g=9.8 \mathrm{~ms}^{-2}$.

$$
\tilde{\lambda}^{2}=\operatorname{diag}\left(\begin{array}{lllll}
0 & 8.32 & 17.57 & 26.39 & 35.19
\end{array}\right) \times 10^{-2} .
$$

\subsection{Natural vibration}

Based on the above parameters, we obtain the natural frequencies and the corresponding natural modes of the system and the shaft deformation factors as listed in Table 1. In these modes, the first one with a zero frequency is a constant pressure mode of the water for the interaction system. As indicated by the data above, in the frequencies of three subsystems: water, hull and shaft, the frequencies of the water domain are lowest and the shaft ones are highest, while the hull ones are in the middle. Therefore, in the frequencies of the integrated interaction system given in Table 1, the first 5 , middle 5 and last 5 ones are near to the ones of water domain, hull and shaft substructures, respectively. As shown in Fig. 7, the mode 8 of non-dimensional frequency 1.0106 corresponds to the hull first elastic mode while the mode 11 of frequency 10.2291 is near to the shaft first elastic bending mode for which the corresponding the shaft frequency factors are calculated in Table 1. For the mode 11, the shaft frequency factor is 1.365 near to the frequency of integrated mode 11 , and the corresponding shaft relative motion factor also takes a big value, which results that in this integrated mode of the system, there exists very large deformation of the shaft. It is also shown the big values of shaft relative motion factors for modes $12 \sim 15$ but the frequency factors are very low, so that the shaft deformation is not large. Since the natural frequency is higher than 37 , the base motions are quite low as indicated in Fig. 8. 
Table 1. Non-dimensional natural frequencies, shaft deformation factors and shaft first elastic

543 frequency factors

\begin{tabular}{cccc}
\hline $\begin{array}{c}\text { Mode number } \\
\text { I }\end{array}$ & $\begin{array}{c}\text { Natural frequency } \\
\hat{\Omega}_{I}\end{array}$ & $\begin{array}{c}\text { Shaft relative motion } \\
\text { factor } \gamma_{I}\end{array}$ & $\begin{array}{c}\text { Shaft frequency } \\
\text { factor } \Omega_{1} / \widehat{\Omega}_{I}\end{array}$ \\
\hline 1 & 0.0000 & $1.86 \mathrm{e}-15$ & $\infty$ \\
2 & 0.0062 & $2.23 \mathrm{e}-08$ & 2252 \\
3 & 0.0900 & $4.65 \mathrm{e}-06$ & 155.1 \\
4 & 0.1073 & $6.60 \mathrm{e}-06$ & 130.1 \\
5 & 0.1324 & $1.00 \mathrm{e}-05$ & 105.5 \\
6 & 0.2691 & $4.15 \mathrm{e}-05$ & 51.89 \\
7 & 0.4858 & $1.35 \mathrm{e}-04$ & 28.74 \\
8 & 1.0106 & $5.89 \mathrm{e}-04$ & 13.82 \\
9 & 2.7567 & $4.50 \mathrm{e}-03$ & 5.065 \\
10 & 5.4032 & $2.00 \mathrm{e}-02$ & 2.584 \\
11 & 10.2291 & $2.21 \mathrm{e}+01$ & 1.365 \\
12 & 37.8349 & $1.86 \mathrm{e}+03$ & 0.369 \\
13 & 53.9887 & $1.01 \mathrm{e}+03$ & 0.259 \\
14 & 56.0603 & $2.96 \mathrm{e}+03$ & 0.249 \\
15 & 79.7987 & $1.453+03$ & 0.175 \\
\hline
\end{tabular}

544

545

546

547
Shaft
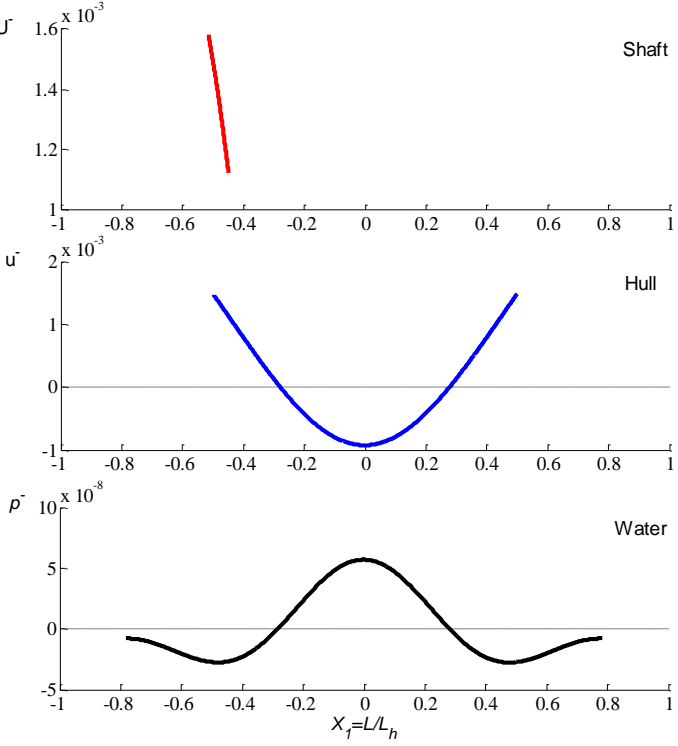

(a)

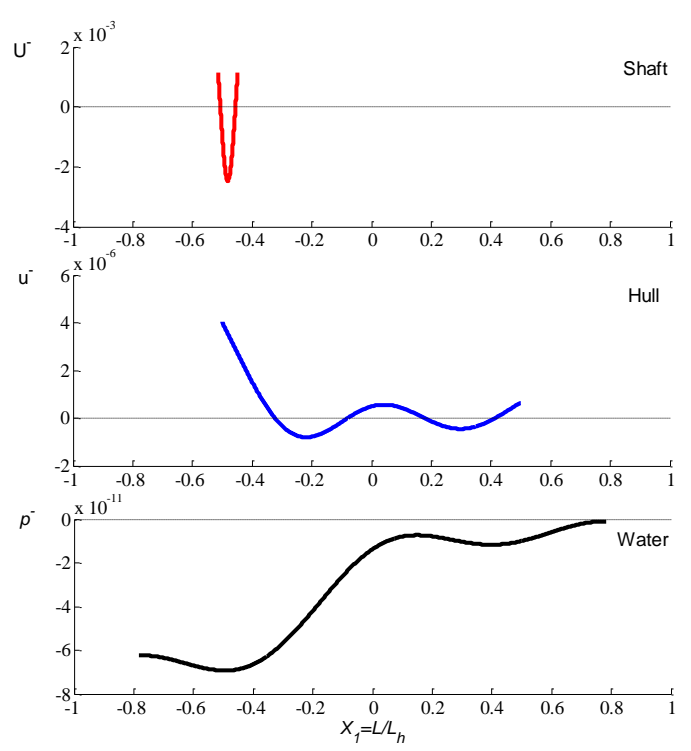

(b)

Fig. 7. The 8-th natural mode of $f_{8}=1.0106$ (a) where the hull is in its first elastic bending form and the 11-th natural mode of $f_{11}=10.2291(\mathrm{~b})$ where the shaft is in its first elastic bending

548 deformation. 


\subsection{Dynamic response}

The main energy of sea waves is located in frequency range lower than $10 \mathrm{~Hz}[24,25]$. To investigate the dynamic response of the integrated system, we consider the sea wave frequency $\breve{\omega}=2 \pi \times(0.5 \sim 10 \mathrm{~Hz})$ for the dynamic response analysis. To investigate the dynamic response characteristics affected by the wave frequency and the bearing stiffness, we define the following nondimensional parameters

Wave frequency

Bearing stiffness parameter

$$
\eta_{f}=\breve{\omega} / \omega
$$$$
\eta_{b}=\omega_{b} / \omega
$$

where $\omega_{b}$ denotes the frequency of the rigid shaft supported by the two baring springs. Taking $\mathrm{dB}$ values referencing to $10^{-12}$ we obtain the following figures to characterise the dynamic behaviour of the system. Fig. 8 shows the dynamic response surface of the shaft base motion with respect to nondimensional wave frequency $\eta_{f}$ and bearing stiffness parameter $\eta_{b}$, while Fig. 9 provides the corresponding dynamic response surface of shaft relative motion factor in Eq. (69). From these surfaces, it is observed that with varying of bearing stiffness parameter there are different dynamic response peaks. To observe these peaks more clearly, Figs. 10 and 11 respectively shows the curves of shaft relative motion factor (69) and twist factor (70) for the case of bearing stiffness parameter $\eta_{b}=0.9$. Fig. 10 shows the maximum peak of the shaft relative motion is 10.23 corresponding the shat first bending deformation, mode 11 in Table 1 and its mode shape shown by the right figure in Fig. 7. Fig. 11 shows the shaft twist peaks at frequencies 1.011 and 10.23 , which correspond to the hull first bending mode and the shaft first bending mode shown in Fig. 7, respectively. The calculated results demonstrate that the defined shaft motion parameters can provide useful information for safety shaft design in huge ships. 


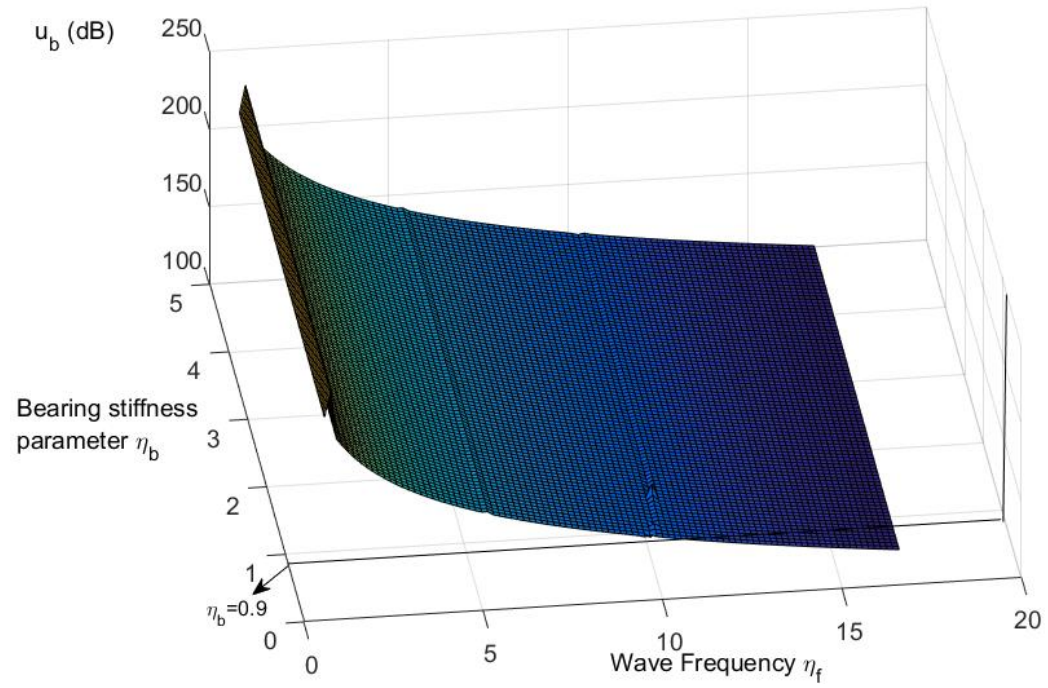

572 Fig. 8. The dynamic response surface of shaft base motion with respect to bearing stiffness parameter

573 and wave excitation frequency.

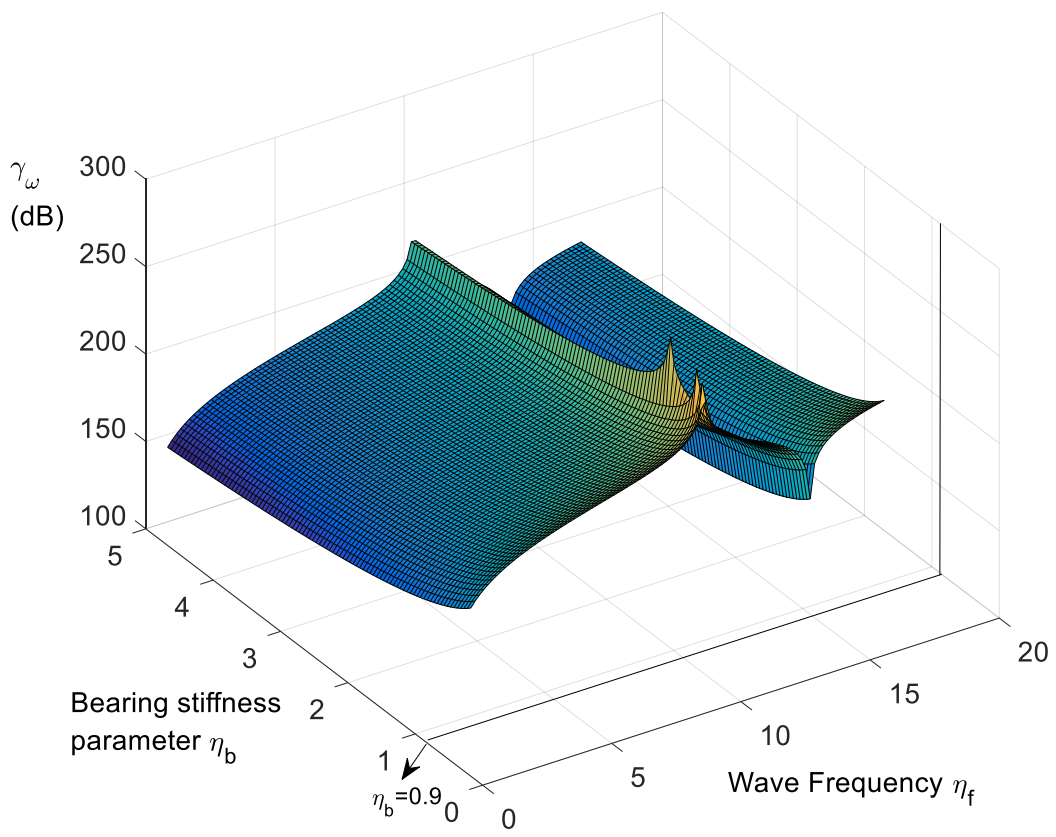

575 Fig. 9. The dynamic response surface of shaft relative motion factor (69) with respect to bearing 576 stiffness parameter and wave excitation frequency. 


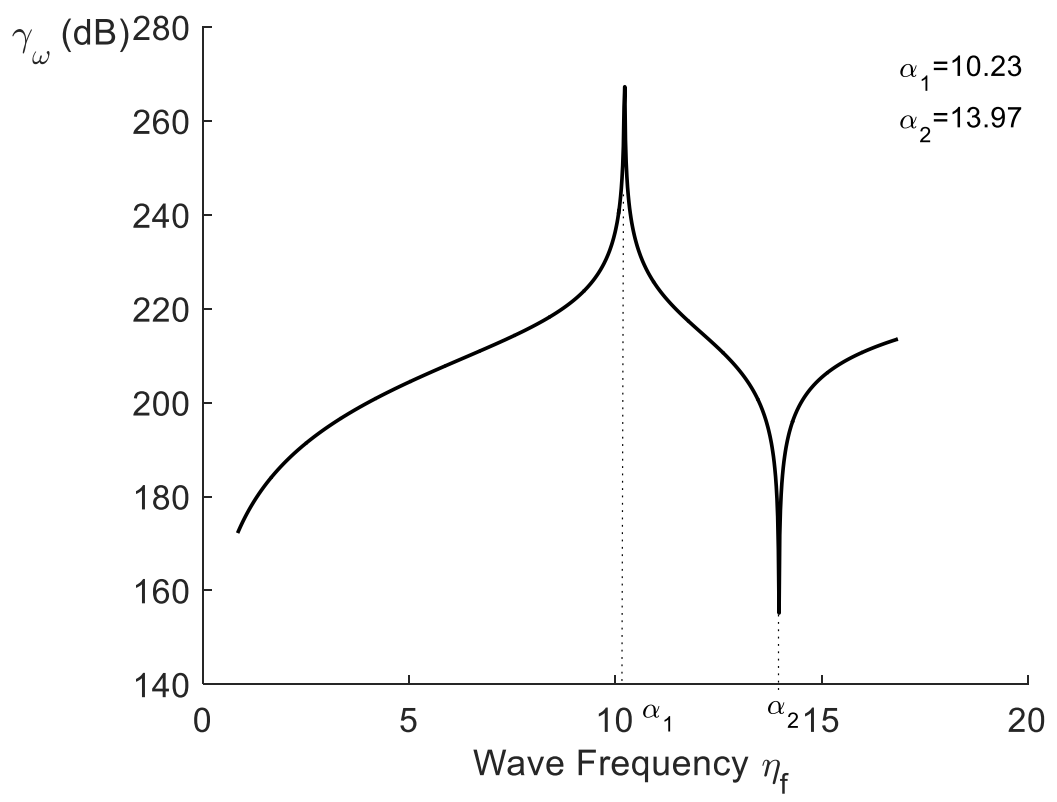

578 Fig. 10. The dynamic response curve of shaft relative motion factor (69) in the case of $\eta_{b}=0.9$.

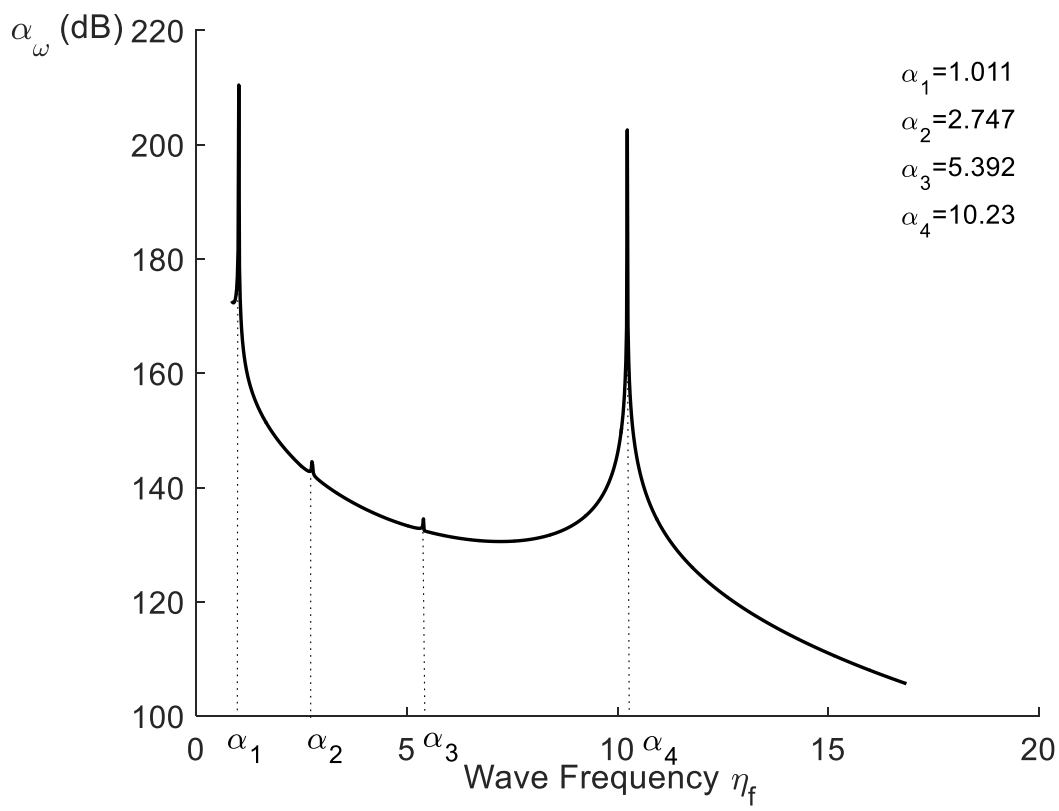

580 Fig. 11. The dynamic response curve of shaft twist factor (70) in the case of $\eta_{b}=0.9$.

\section{Conclusion \& discussion}

The developed integrated theory and the corresponding numerical model can provide a useful mean for large ship - propulsion system designs considering the engine safety operations. As an example, a

584 2-D numerical model gives the numerical results to illustrate the applications of the proposed 585 numerical approach, which can be extended to the complex case for practical designs. 
587 the natural vibrations of the integrated system, the shaft frequency factor given by Eq. (56) should be

588 far from 1, and the shaft deformation factor given by Eq. (60) should be small to avoid large shaft motion and deformation in each integrated mode. The first bending frequency of the hull and the shaft may play important role in dynamic designs. For the large ships, its first bending frequency will be small which may not be avoided. It would be beneficial to reduce the shaft dynamic response if designing a higher shaft bending frequency. The suggested dynamic design may take the three steps: 1) initial design using a 2-D model as example to choose initial parameters; 2) middle design using the designed data based on the first step to undergo 3-D analysis using the proposed model to modify the design data; 3) final check and modification by the analysis of natural vibrations and dynamic responses.

The proposed model has not very detailed considered the effects of ships with its moving speed, although it is assumed as a stationary motion on which disturbance vibrations are interested in this paper. A further investigation on this case to explore some effect may be carried on in future.

\section{Acknowledgements}

Authors acknowledge NSFC to support this research by a key project: No. 51139005. Thanks are also given to WHUT for supporting J.T. Xing as a visiting professor to engage the joint research in WHUT and for awarding a studentship (WUT: 2014-JL-006) for Z. Tian to study in the University of Southampton to carry on this collaboration research for one year.

605

\section{References}

Bishop, R.E.D. and Price, W.G. Hydroelasticity of ships. Cambridge University Press, London, 1979.

Bishop, R.E.D., Price, W.G. and Wu, Y., 1986. A general linear hydroelasticity theory of floating structures moving in a seaway. Phil. Trans. R. Soc. Lond. A. 316, 375-426.

Bernhard B. Rotordynamics: Unit 4 - Modeling Journal Bearings, Mech. Des. Res. Lab, Mech. Eng. - Eng. Mech. Dept., Michigan Technol. Univ. Web site: http://www.me.mtu.edu/ mdrl.

Bathe K.J. Finite Element Procedures in Engineering Analysis, Prentice-Hall, New Jersey, 1996.

Craig, R.R. and Bampton, M.C.C.,1968. Coupling of substructures for dynamical analysis. AIAA. Jl. 6(7), 1313-1319. 
Dymarski, C., 2009. Analysis of ship shaft line coupling bolts failure, J. Pol. Cimac. 4, 33-40.

Deruieux, A. Fluid-Structure Interaction, Kogan Page Limited, London, 2003.

Fonte, M., Freitas, M., 2009. Marine main engine crankshaft failure analysis: A case study. Eng. Fail. Anal. 16, 1940-1947.

Fung, Y.C. A First Course in Continuum Mechanics. 1977.

Jun, M., Kazunobu, F., Kazuhide, O., Kazuyuki, A., Koji, K., 1998. Study on Dynamic Interaction between Ship Hull and Main Engine Structure (in Japanese). J. Soc. Naval Archit. Japan. 184, 393 400

Leontopoulos, C., 2006. Shaft alignment and powertrain vibration. ABS.

Li, Z. X., Yan, X. P., Qin, L., Chen K. and Xing, J. T., 2015. Robust global sliding model control for water-hull-propulsion unit interaction systems Part 1: system boundary identification. Tehnicki Vjesnik-Tech. Gazette. 22, (2), 465-473.

Li, Z. X., Yan, X. P., Qin, L., Chen K. and Xing, J. T., 2015. Robust global sliding model control for water-hull propulsion unit interaction systems Part 2: model validation. Tehnicki Vjesnik-Tech. Gazette. 22(1), 209-215.

Li, Z.X. Investigation on the Dynamics Modelling and Condition Monitoring of the Propulsion System in Large Scale Ships (Ph. D Thesis). Wuhan Univ. Technol., 2013.

Lu J., 2010. The effects of wave loads on ship propulsion systems, J. Shanghai Jiaotong Univ. 44, 4091413.

Li, S., 2009. A progress review on VIRTUE planed research. J. Ship Mech. 13, 662-675.

Murawski, L., 2005. Shaft line alignment analysis taking ship construction flexibility and deformations into consideration. Mar. Struct. 18, 62-84.

Moctar O.EI., Junglewitz A., 2005. New attitudes needed for giant container ships. Naval Archit. 4-9.

Murawski, L., Charchalis, A., 2015. An estimation method for the torsional vibration of the marine propulsion system. Solid State Phenom. 220-221, 71-80.

Morand, H.J.P. and Ohayon, R. Fluid structure interaction. John Wiley and Sons, Chichester, 1995.

Newman, J.N., 1978. The Theory of Ship Motions. ADV APPL MECH. 18, 221-283. 
Ogawa Y., 2011. A whole ship finite element analysis with the input of nonlinear wave loads in the irregular and multi-directional waves. ASME 2011 30th International Conference on Ocean, Offshore and Arctic Engineering, 19-24 June. Netherlands.

Pouw, C.P., 2008. Development of a multi-objective design optimization procedure for marine propellers (Master Thesis). Delft Univ. Technol.

Roemen, R., Grevink, J., 2009. An advanced approach to the design of shaft-lines and bearing arrangements for fast ferries. Wartsilia Tech. J. 1, 47-53.

Shi, L., 2010. Research on shafting alignment considering ship hull deformations. Mar. Struct. 23, $103-114$.

Shaft alignment: rigid shafting and flexible hulls, 2000. The Motor Ship

Schulten P., 2005. The Interaction between diesel engine, ship and propeller during manoeuvring. Delft Univ. Technol.

The Swedish Club Highlights, 2005.

Tang, B., Brennan, M. J., 2013. On the influence of the mode-shapes of a marine propulsion shafting system on the prediction of torsional stresses. J. Mar. Sci. Technol. 2, 209-214.

Tian, Z., Yan, X.P., Li Z.X. and Zhang C., 2014. Dynamic interaction analysis of a 2D propulsion shaft-ship hull system subjected by sea wave. Proceedings of the International Conference on Offshore Mechanics and Arctic Engineering, 6-8 July. USA, v 4A.

Tu, H. and Chen H., 2014. Modeling and simulation of a large marine diesel propulsion system. Shipbuild. China. 55, 158-167.

Tan, M., Xiong, Y.P., Xing, J.T. and Toyoda, M.., 2006 A numerical investigation of natural characteristics of a partially filled tank using a substructure method. In Proceedings of Hydroelasticity 2006: Hydroelasticity in Marine Technology. pp. 181-190.

Xing, J.T. and Price, W.G., 1991. A mixed finite element method for the dynamic analysis of coupled fluid-solid interaction problems. Proc. R. Soc. Lond. A. 433, 235-255.

Xing, J.T., Price, W.G. and Du, Q.H., 1996. Mixed finite element substructure-subdomain methods for the dynamical analysis of coupled fluid-solid interaction problems. Phil. Trans. R. S. Lond. A. 354, 259-295. 
Xing, J.T., Xiong, Y.P. and Tan, M., 2009. Developments of a mixed finite element substructuresubdomain method for fluid-structure interaction dynamics with applications in maritime engineering. P I MECH ENG M-J ENG. 223 (3), 399-418.

Xing, J.T. Theoretical manual of fluid-structure interaction analysis program-FSIAP. Univ. Soton. 1995.

Xing, J.T. User manual fluid-structure interaction analysis program-FSIAP. Univ. Soton. 1995.

Xing, J.T., Price, W.G. and Wang, A., 1997. Transient analysis of the ship-water interaction system excited by a pressure water wave. Mar. Struct. 10(5), 305-321.

Xing, J.T. Xiong, Y.P., Tan, M. and Toyota, M., 2006. Vibration problem of a spherical tank containing jet propellant: numerical simulations. Ship Science Report No. 141, ISSN0140-3818.

Xiong, Y.P., Xing, J.T. and Tan, M., 2006. Transient dynamic responses of an internal liquid-LNG tank-sea water interaction system excited by waves and earthquake loads, In Proceedings of the 14th International Congress on Sound and Vibration, July. Cairns, Australia, Paper number 566, pp.1-8.

Xiong, Y.P., Xing, J.T. and Price, W.G., 2006. The interactive dynamic behaviour of an air-liquidelastic spherical tank system. In Proceedings of 2006 ASME Pressure Vessels and Piping Division Conference, Vancouver, 23-27 July. BC, Canada, pp. 1-8.

Xing, J.T., Xiong, Y.P. and Tan, M., 2007. The natural vibration characteristics of a water-shell tank interaction system. In Advancements in Marine Structures, Proceedings of Marstruct 2007, 1st International Conference on Marine Structures, 12-14 March. Glasgow, UK, pp. 305-312.

Xing, J.T., Xiong, Y.P. and Tan, M., 2007. The dynamic analysis of a building structure - acoustic volume interaction system excited by human footfall impacts, In Proceedings of Fourteenth International Congress on Sound and Vibration, 9-12 July. Cairns, Australia, Paper number 147.

Xiong, Y.P. and Xing, J.T., 2007. Natural dynamic characteristics of an integrated liquid - LNG tank - water interaction system. In Advancements in Marine Structures, Proceedings of Marstruct 2007, 1st International Conference on Marine Structures, 12-14 March. Glasgow, UK, pp. 313-321. 
Xing, J.T. and Xiong, Y.P., 2008. Numerical simulations of a building-acoustic volume interaction system excited by multiple human footfall impacts. In Proceedings of 2008 ASME Pressure Vessels and Piping Division Conference, July 27-31. Chicago, Illinois, PVP2008-61813, pp. 1-10.

Xing, J.T. and Xiong, Y.P. Mixed finite element method and applications to dynamic analysis of fluidstructure interaction systems subject to earthquake, explosion and impact loads. In Proceedings of ISMA 2008 International Conference on Noise and Vibration Engineering,15-17 September, 2008. Leuven, Belgium, Paper ID-562, pp.1-15.

Xiong, Y.P. and Xing, J.T., 2008. Dynamic analysis and design of LNG tanks considering fluid structure interactions, In Proceedings of 27th international conference on offshore mechanics and arctic engineering, 15-20 June. Estoril, Portugal, OMAE2008-57937, pp. 1-8.

Xiong, Y.P. and Xing, J.T., 2008. Transient dynamic responses of an integrated air-liquid-elastic tank interaction system subject to earthquake excitations. In 2008 ASME Pressure Vessels and Piping Division Conference-PVP2008, 27-31 July. Chicago, Illinois, PVP2008-61815, pp.1-10.

Xing, J.T., 2007. Natural vibration of two-dimensional slender structure-water interaction systems subject to Sommerfeld radiation condition. J. Sound Vib. 308, 67-79.

Xing, J.T., 2008. An investigation into natural vibrations of fluid-structure interaction systems subject to Sommerfeld radiation condition. Acta Mech Sin. 24, 69-82.

Xing, J.T., 1986. A study on finite element method and substructure-subdomain technique for dynamic analysis of coupled fluid-solid interaction problems. Acta Mechanica Solida Sinica. 4, 329-337.

Xing, J.T., 1986. Mode synthesis method with displacement compatibility for dynamic analysis of fluid-solid interaction problems. Acta Mech. Solida Sin. 7, 148-156.

Xing, J.T. and Zheng, Z.C.,1983. A study upon mode synthesis methods based on variational principles for elastodynamics. Acta Mech. Solida Sin. 2, 250-257.

Yan, X.P., Li, Z.X., Liu, Z.L., Yang, P., Zhu, H.H., Yang, Z.M., 2013. Study on coupling dynamical theory for interaction of propulsion system and hull of large ships: a review. J. Ship Mech. 4, 439449.

Zienkiewicz, O.C. and Taylor, R.L. The Finite Element Method. McGraw-Hill, New York. 4th edition, 1991. 